\title{
Different dose regimes and administration methods of tranexamic acid in cardiac surgery: a meta-analysis of randomized trials
}

Jingfei Guo ${ }^{1}$, Xurong Gao ${ }^{2}$, Yan Ma ${ }^{3}$, Huran Lv' ${ }^{1}$ Wenjun Hu${ }^{4}$, Shijie Zhang ${ }^{5}$, Hongwen Ji', Guyan Wang ${ }^{1}$ and Jia Shi ${ }^{*}$ (D)

\begin{abstract}
Background: The efficacy of tranexamic acid (TXA) to reduce perioperative blood loss and allogeneic blood transfusion in cardiac surgeries has been proved in previous studies, but its adverse effects especially seizure has always been a problem of concern. This meta-analysis aims to provide information on the optimal dosage and delivery method which is effective with the least adverse outcomes.

Methods: We searched Cochrane Central Register of Controlled Trials, MEDLINE and EMBASE for all relevant articles published before 2018/12/31. Inclusion criteria were adult patients undergoing elective heart surgeries, and only randomized control trials comparing TXA with placebo were considered. Two authors independently assessed trial quality and extracted relevant data.

Results: We included 49 studies with 10,591 patients into analysis. TXA significantly reduced transfusion rate (RR 0.71, 95\% Cl 0.65 to $0.78, \mathrm{P}<0.00001)$. The overall transfusion rate was 35\%(1573/4477) for patients using TXA and 49\%(2190/ 4408) for patients in the control group. Peri-operative blood loss (MD $-246.98 \mathrm{ml}, 95 \% \mathrm{Cl}-287.89$ to $-206.06 \mathrm{ml}$, $\mathrm{P}<0.00001$ ) and re-operation rate (RR $0.62,95 \% \mathrm{Cl} 0.49$ to $0.79, \mathrm{P}<0.0001$ ) were also reduced significantly. TXA usage did not increase risk of mortality, myocardial infarction, stroke, pulmonary embolism and renal dysfunction, but was associated with a significantly increase in seizure attack (RR 3.21, 95\% Cl 1.04 to $9.90, P=0.04$ ). The overall rate of seizure attack was $0.62 \%(21 / 3378)$ for patients using TXA and 0.15\%(5/3406) for patients in the control group.

In subgroup analysis, TXA was effective for both on-pump and off-pump surgeries. Topical application didn't reduce the need for transfusion requirement, while intravenous delivery no matter as bolus injection alone or bolus plus continuous infusion were effective. Intravenous high-dose TXA didn't further decrease transfusion rate compared with low-dose regimen, and increased the risk of seizure by 4.83 times. No patients in the low-dose group had seizure attack.
\end{abstract}

Conclusions: TXA was effective in reducing transfusion requirement in all kinds of cardiac surgeries. Low-dose intravenous infusion was the most preferable delivery method which was as effective as high-dose regimen in reducing transfusion rate without increasing the risk of seizure.

Keywords: Tranexamic acid, Cardiac surgery, Seizure, Dose regimen

\footnotetext{
* Correspondence: shjfuwai@sina.com

Jingfei Guo, Xurong Gao and Yan Ma are co-first authors of this paper, and

Jia Shi is the corresponding author

${ }^{1}$ Department of Anesthesiology, Fuwai Hospital, National Center for

Cardiovascular Diseases, Chinese Academy of Medical Sciences, and Peking

Union Medical College, No.167 Beilishi Road, Xicheng district, Beijing, China

Full list of author information is available at the end of the article
}

(c) The Author(s). 2019 Open Access This article is distributed under the terms of the Creative Commons Attribution 4.0 International License (http://creativecommons.org/licenses/by/4.0/), which permits unrestricted use, distribution, and reproduction in any medium, provided you give appropriate credit to the original author(s) and the source, provide a link to the Creative Commons license, and indicate if changes were made. The Creative Commons Public Domain Dedication waiver (http://creativecommons.org/publicdomain/zero/1.0/) applies to the data made available in this article, unless otherwise stated. 


\section{Background}

Tranexamic acid(TXA)is a lysine analogues which acts principally by blocking the lysine binding sites on plasminogen molecules, inhibiting the formation of plasmin and therefore inhibiting fibrinolysis [1]. The efficacy of TXA to reduce perioperative blood loss and allogeneic blood transfusion has been studied extensively. The latest Cochrane review on TXA was published in 2011 [2], the author concluded that TXA was effective in reducing blood loss and transfusion requirement in multiple kinds of surgeries including cardiac surgery, and appeared to be free of serious adverse effects. However, as an anti-fibrinolytic agent, the prothrombotic effect has always been a concern. TXA could potentially increase the risk of myocardial infarction, stroke, and other thrombotic complications, and has later been shown to increase the risk of neurologic events [3, 4], especially seizures [5].

In 2017, Myles et al. published a study on TXA usage in cardiac surgery [6]. In this randomized controlled trial including 4631 patients, the author found that seizures occurred in 0.7 and $0.1 \%$ in TXA and placebo group respectively $(P=0.002)$. This recent study draws further attention to the dosage regimen of TXA, since adverse effects including seizure are possibly dosagerelated. The study used a high-dose regimen, in which either $50 \mathrm{mg} / \mathrm{kg}$ or $100 \mathrm{mg} / \mathrm{kg}$ of TXA was delivered for each patient. There is a possibility that lower dose of TXA can be equally effective while causing less adverse effects. In fact, TXA plasma concentrations required to suppress fibrinolysis and plasmin-induced platelet activation are merely 10 and $16 \mu \mathrm{g} / \mathrm{ml}$, respectively $[7,8]$. This relatively low plasma concentration can be reached in cardiac surgery when $10 \mathrm{mg} / \mathrm{kg}$ of TXA is administered as a bolus then followed by continuous infusion of $1 \mathrm{mg} \mathrm{kg} / \mathrm{h}$ and $1 \mathrm{mg} / \mathrm{kg}$ in CPB [9]. But another potential mechanism of TXA action might be the increase in thrombin formation, which requires concentrations more than $126 \mu \mathrm{g} / \mathrm{ml}$ to be effective [10, 11]. $30 \mathrm{mg} / \mathrm{kg}$ of TXA administered as a bolus followed by $16 \mathrm{mg} / \mathrm{kg} / \mathrm{h}$ and $2 \mathrm{mg} / \mathrm{kg}$ in CPB prime solution was able to maintain the plasma concentration above $114 \mu \mathrm{g} / \mathrm{ml}[9]$.

The dosage and delivery methods of TXA in cardiac surgery has long been a problem of debate. Drug administration varied significantly across studies, and no agreement has been reached concerning the following issues: what is the ideal dosage of TXA, whether TXA should be delivered intravenously or topically, whether continuous infusion or bolus injection should be used. This study aims to summarize evidence from all trials published before 2019 and try to provide information on the optimal dosage and delivery method which is effective with the least adverse outcomes.

\section{Methods}

\section{Criteria for considering studies for this review-the PICO framework \\ Patient}

Adults who underwent elective heart surgery were included in the study. Trials on urgent cases or on children were excluded. We included both on and off pump operations. (sentence removed).

\section{Intervention}

Tranexamic acid (TXA) was the intervention in this study. Both topical and intravenous application of TXA were considered.

\section{Comparison}

Only randomized controlled trials(RCTs)comparing TXA with placebo were included in this meta-analysis. Head to head trials comparing TXA with other fibrinolytic medications were excluded from analysis.

\section{Outcome}

Primary outcomes were transfusion rate (the proportion of patients underwent blood transfusion during hospital stay) and transfusion volume (the amounts of blood transfused during hospital stay). Secondary outcomes were post-operative blood loss, re-operation rate, mortality during hospital stay, post-operative complications, including seizure, stroke, myocardial infarction, pulmonary embolism and renal dysfunction.

\section{Search methods for identification of studies Electronic searches}

MEDLINE, EMBASE, and the Cochrane Central Register of Controlled Trials (CENTRAL) were searched systematically for relevant studies. We did not limit the search by language or publication state. Searches in all three databases were searched until 2018/12/31. The methodology was developed from the Preferred Reporting Items for Systematic Reviews and Meta-Analyses (PRISMA) statemen. Details for search strategies were listed in Additional file 1.

\section{Searching other resources}

We used Google (including Google Scholar) to do comprehensive research on the Internet. We also scanned the reference lists of identified articles and related reviews for relevant studies.

\section{Data collection and analysis Study selection}

The titles and abstracts identified in the electronic search were independently screened by two authors to identify trials that met previously defined inclusion criteria. 


\section{Data extraction and management}

Two authors independently extracted study characteristics and outcomes. Data on the following items were recorded: type of surgery, usage of cardiopulmonary bypass (CPB), presence or absence of a transfusion protocol, method of TXA administration (topical or intravenous, continuous or bolus), drug dosage, number of patients in the experiment and control group, and the outcome measures of interest. The discrepancy was resolved by discussion.

\section{Risk of bias assessment}

All included studies were assessed for methodological quality by two authors independently. We used the Cochrane Collaboration's tool for assessing risk of bias (Version 5.1.0) as evaluation criteria. The following domains were assessed for each study: random sequence generation, allocation concealment, blinding of participants and personnel, blinding of outcome assessment, incomplete outcome data addressed and selective reporting. Each domain for every included study was graded into three categories: low risk of bias, unclear risk of bias and high risk of bias.

\section{Subgroup analysis and investigation of heterogeneity}

Analysis of a-priori subgroups was performed according to the following factors: type of surgery, usage of cardiopulmonary bypass $(\mathrm{CPB})$, intravenous or topical application of TXA, bolus or continuous infusion of TXA, and TXA dosage.

\section{Statistical analysis}

For dichotomous outcomes (transfusion rate, re-operation rate, incidence of adverse effects), we calculated relative risk (RR) with 95\% confidence interval (CI). For continuous outcomes (post-operative blood loss or transfusion volume) reported as mean and standard deviation, the mean difference (MD) for the pooled estimates with $95 \% \mathrm{CI}$ was calculated. The fixed-effect model was used for analysis with no heterogeneity $\left(\mathrm{I}^{2}=0\right)$, and random-effect model was used for analysis with heterogeneity $\left(\mathrm{I}^{2}>0 \%\right)$. For outcomes with heterogeneity, an effort was made to identify its source, mainly through subgroup analysis. A $P$-value less than or equal to 0.05 for the $\mathrm{Q}$ statistic was used to define statistically significant heterogeneity. Statistical heterogeneity was also assessed using the $\mathrm{I}^{2}$ test. $\mathrm{I}^{2}=0-40 \%$ : heterogeneity might not be important; $\mathrm{I}^{2}=30-60 \%$ : may represent moderate heterogeneity; $\mathrm{I}^{2}=50$ to $90 \%$ : may represent substantial heterogeneity; $\mathrm{I}^{2}=75$ to 100\%: considerable heterogeneity. $P<0.05$ (2-sided) was considered statistically significant for the hypothesis testing. The publication bias was visualized by symmetry of funnel plot. All statistical analyses were performed in RevMan (version5.0; Cochrane Collaboration, Oxford, UK) and Stata (version 9.0; Stata Corporation, College Station, TX).

\section{Results \\ Description of studies \\ Search results}

We identified 4541 results according to the search strategy described above. The detailed search process was provided in Additional file 1. After assessing 103 full-text articles for eligibility, 49 of them were finally included in the metaanalysis [6, 12-59] (Fig. 1).Major exclusions were provided in Additional file 2.

\section{Included studies}

We included 49 studies with a total of 10,591 participants in this meta-analysis. The detailed characteristics of all included studies were listed in Additional file 3 One thing worth noticing was that intravenous drug administration varied significantly between trials on dosage regimen. There were mainly two types of intravenous administration methods. One was bolus infusion alone (14 trials) and the other was bolus injection followed by continuous infusion (22 trials). Since these two delivery methods varied significantly in pharmacokinetics, we decided the cutoff value for high and low dose TXA separately. According to what's mentioned in the background, we defined $<50 \mathrm{mg} / \mathrm{kg}$ of TXA as low dose for bolus injection, and $\leq 10 \mathrm{mg} / \mathrm{kg}+1 \mathrm{mg} / \mathrm{kg} / \mathrm{h}$ TXA as low dose for bolus plus continuous infusion.

\section{Risk of bias in included studies}

Details regarding the performance of the studies against each domain were presented in the Risk of bias graph (Fig. 2). Additionally, a visual summary of judgement $s$ about each methodological quality item for each included trial was shown in Fig. 3.

Most of the trials included had low risk of bias on random sequence generation, blinding, incomplete outcome data and selective reporting. Selective reporting turned out to be the main risk of bias in this meta-analysis. We rated 33 trials as having low risk of selective reporting, 11 trials as having high risk of selective reporting and 5 trial as having unclear risk of selective reporting. Typical bias for selective reporting was that some studies failed to report transfusion rate while only reporting transfusion volume or vice versa. There was a risk that insignificant data were concealed, and results were thus misrepresented. Also, many of the trials failed to report information on allocation concealment and were rated to be have unclear risk of bias for this item. In summary, there were 13 trials with high risk of bias, 13 trials with low risk of bias and 23 studies with unclear risk of bias.

\section{Effects of interventions}

Effects and adverse outcomes

\section{Transfusion rate}

There were 31 trials with 8925 patients that reported data on the number of patients exposed to blood transfusion. 


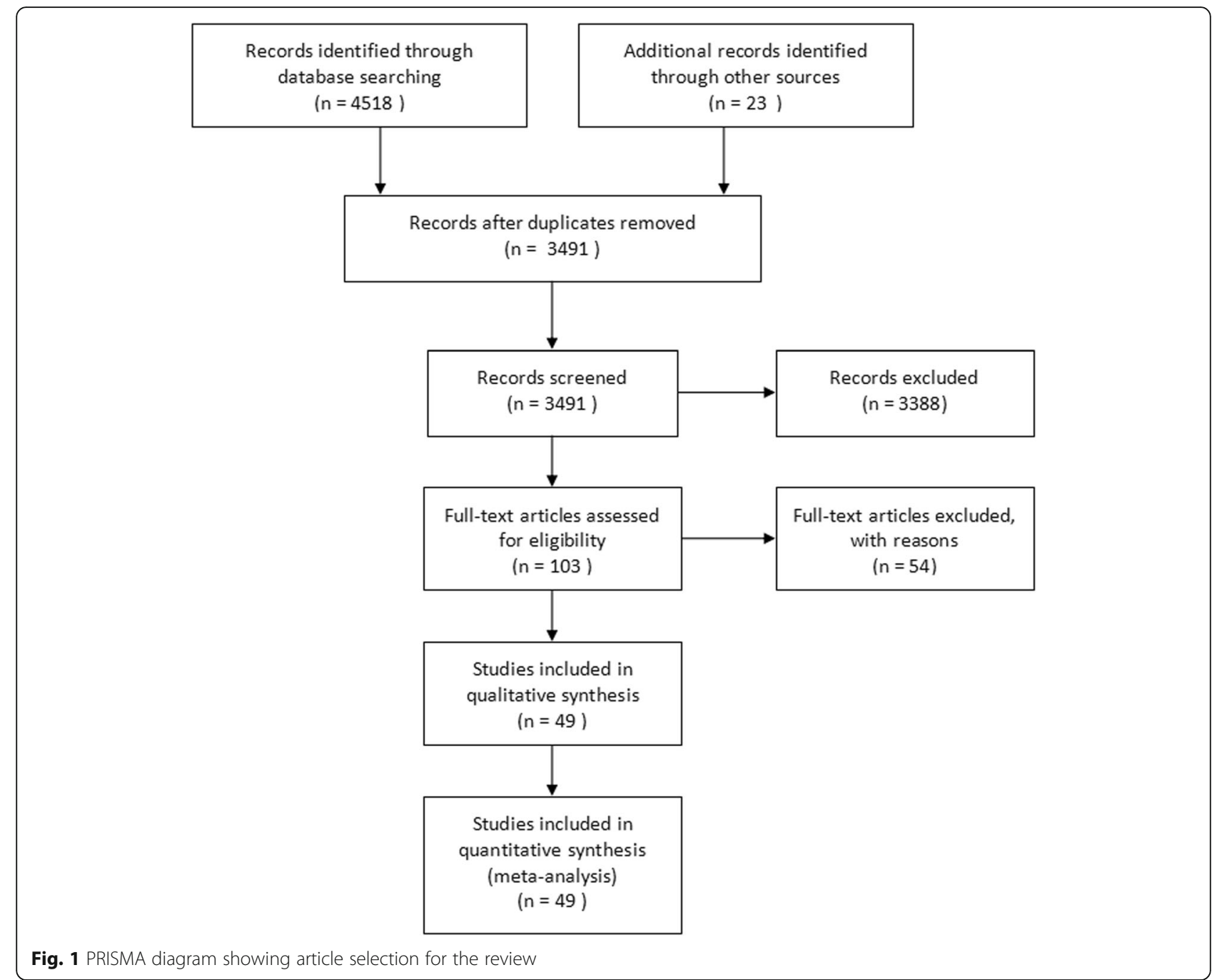

The use of TXA significantly reduce the need for allogeneic blood transfusion by a relative $29 \%$ (RR $0.71,95 \%$ CI 0.65 to $0.78, \mathrm{P}<0.00001)$. The overall transfusion rate was $35 \%(1573 / 4477)$ for patients using TXA and 49\%(2190/ 4408) for patients in the control group, with a reduction in absolute risk of 0.14 (95\% CI 0.12, 0.16). Heterogeneity between these trials was moderate $\left(\mathrm{Chi}^{2}=60.98, \mathrm{df}=31\right.$, $\left.P=0.001 ; \mathrm{I}^{2}=49 \%\right)($ Fig. 4).

\section{Transfusion volume}

There were 10 trials with 2105 patients that reported data on the volume of blood transfused in all patients. The use of TXA resulted in 0.6 units reduction of allogeneic blood per patient (MD-0.60 units, $95 \% \mathrm{CI}-0.85$ to -0.35 unit, $\mathrm{P}<0.00001)$. Heterogeneity between these trials was significant $\left(\mathrm{Chi}^{2}=117.26, \mathrm{df}=9, \mathrm{P}<0.00001 ; \mathrm{I}^{2}=92 \%\right)$ (Fig. 5a).

There were 14 trials with 6610 patients that provided data on the volume of blood transfused in those patients transfused. The use of TXA resulted in a 1.02 units reduction of blood transfusion per patient (MD - 1.02 units, $95 \% \mathrm{CI}-1.47$ to -0.56 units, $\mathrm{P}<0.00001)$. Heterogeneity between trials was significant $\left(\mathrm{Chi}^{2}=218.12\right.$, $\mathrm{df}=13, \mathrm{P}<0.00001 ; \mathrm{I}^{2}=94 \%$ ) (Fig. 5b).

\section{Post-operative blood loss}

There were 44 trials with 5560 patients that reported post-operative blood loss. On average, TXA treatment reduced post-operative blood loss by around $247 \mathrm{ml}$ per patient compared to control (MD $-246.98 \mathrm{ml}$, $95 \%$ CI -287.89 to $-206.06 \mathrm{ml}, \mathrm{P}<0.00001)$. Heterogeneity between these trials was significant $\left(\mathrm{Chi}^{2}=1278.68\right.$, $\mathrm{df}=43, P<0.00001 ; \mathrm{I}^{2}=97 \%$ ) (Fig. 6).

Re-operation There were 32 trials with 8937 patients that reported data on reoperation for all reasons. The use of TXA significantly decrease the risk of reoperation by $38 \%$ (RR $0.62,95 \% \mathrm{CI} 0.49$ to $0.79, \mathrm{P}<0.0001)$. The overall reoperation rate was $2.4 \%(105 / 4472)$ for patients using TXA and 3.9\% 


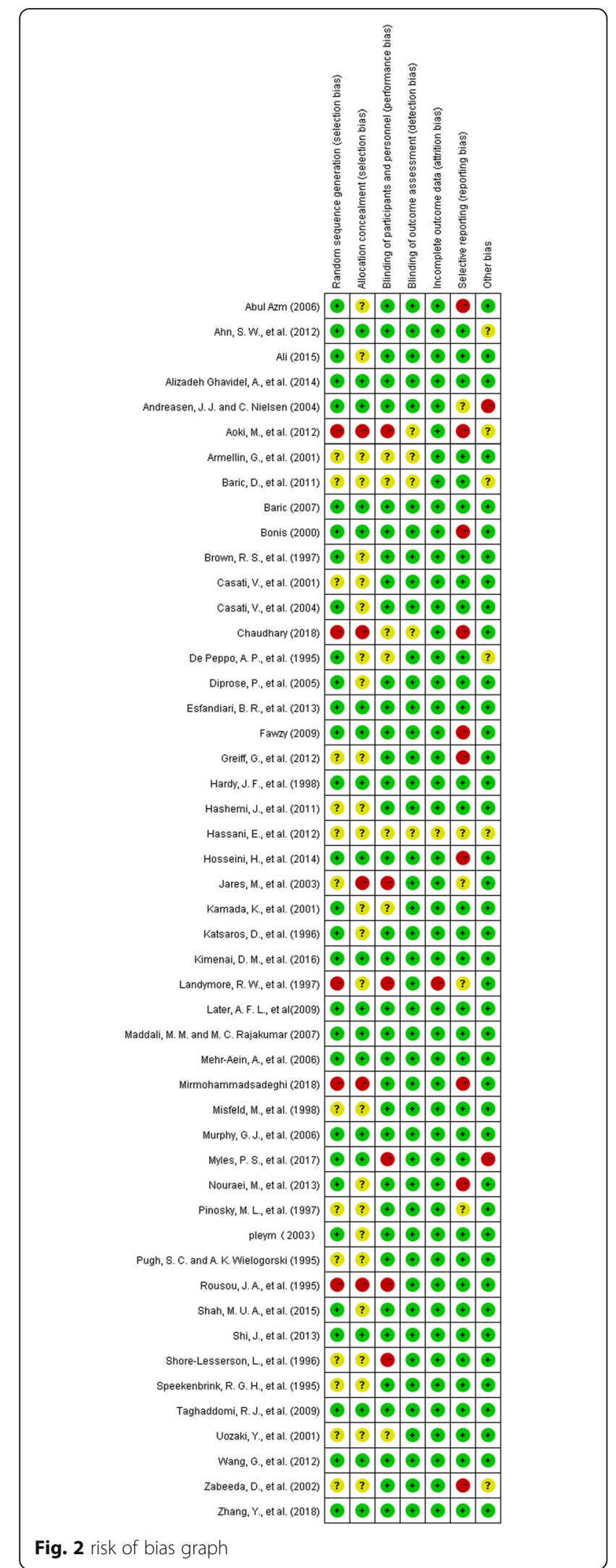

(173/4465) for patients in the control group, with a reduction in absolute risk of 0.01 ( $95 \% \mathrm{CI} 0.01,0.02)$. Heterogeneity between these trials were low $\left(\mathrm{Chi}^{2}=18.86, \mathrm{df}=23, P=0.71\right.$; $\left.\mathrm{I}^{2}=0 \%\right)$ (Fig. 7).

\section{Seizure}

There were 11 trials with 6784 patients that reported data for seizure. The use of TXA was associated with a 3.21 folds increase in the risk of seizure (RR 3.21, 95\% CI 1.04 to 9.90, $P=0.04)$. The overall rate of seizure attack was $0.62 \%(21 /$ 3378 ) for patients using TXA and $0.15 \%(5 / 3406)$ for patients in the control group, with an increase in absolute risk of 0.00 (95\%CI 0.00, 0.01).Mild heterogeneity existed between these trials $\left(\mathrm{Chi}^{2}=3.57, \mathrm{df}=3, P=0.31 ; \mathrm{I}^{2}=16 \%\right)$ (Fig. 8).

\section{Mortality, thrombotic events and renal injury}

As is shown in Table 1, there was no significant difference between TXA and control group in mortality (29 studies, 8970 patients, $P=0.20$ ), stroke (32 studies, 9257 patients, $P=0.50$ ), myocardial infarction (32 studies, 8688 patients, $P=0.67)$, pulmonary embolism (18 studies, 6587 patients, $P=0.60$ ) or renal dysfunction (19 studies, 7210 patients, $P=$ 0.92). All of these results were of low heterogeneity with $\mathrm{I} 2=0 \%$. All the detailed information and forest plot of these results were proved in Additional file 4: Figure S1, Additional file 5: Figure S2, Additional file 6: Figure S3, Additional file 7: Figure S4 and Additional file 8: Figure S5.

\section{Subgroup analysis}

For primary outcomes, subgroup analysis was done only on transfusion rate, given the fact that too few studies reported results on transfusion volume (either for all patients or for transfused patients) to conduct subgroup evaluation.

For secondary outcomes, subgroup analysis was done merely on seizure which was the only adverse event showing significant difference between TXA and control group.

\section{Transfusion rate-—on and off-pump surgeries}

There were 7 off-pump trials and 23 on-pump trials that reported data for transfusion rate. The use of TXA significantly reduce the need for allogeneic blood transfusion by a relative $40 \%$ (RR $0.60,95 \%$ CI 0.44 to $0.83, P=0.002$ ) for off-pump trials and $29 \%$ (RR $0.71,95 \%$ CI 0.63 to $0.80, \mathrm{P}<0.00001$ ) for on-pump trials. Detailed data were provided in Additional file 9: Figure S6.

\section{Transfusion rate-_types of surgery}

There were 22 trials on CABG and 8 trials on other types of heart surgeries that reported data for transfusion rate. The use of TXA significantly reduce the need for allogeneic blood transfusion by a relative $31 \%$ (RR $0.69,95 \%$ CI 0.65 to 0.73 , $\mathrm{P}<0.00001$ ) for CABG trials and $15 \%$ (RR 0.85 , 95\% CI 0.74 


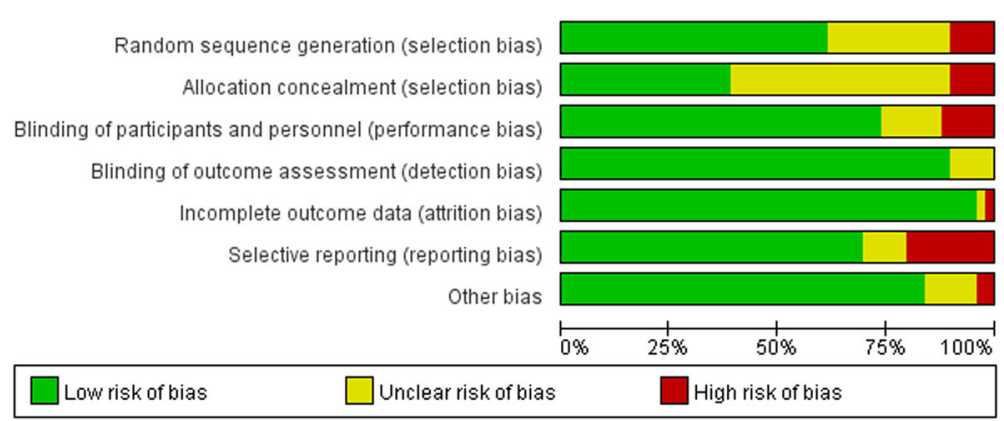

Fig. 3 Risk of bias summary

to $0.96, P=0.01$ ) for trials including other kinds of surgery. Detailed data were provided in Additional file 10: Figure S7.

\section{Transfusion rate-_intravenous and topical application}

There were 28 trials on intravenous (IV) infusion and 4 trials on topical application of TXA that reported data for transfusion rate. For IV infusion, the use of TXA significantly reduce the need for allogeneic blood transfusion by a relative $30 \%$ (RR 0.70 , 95\% CI 0.66 to $0.74, \mathrm{P}<0.00001$ ). Heterogeneity between these trials was moderate $\left(\mathrm{Chi}^{2}=\right.$ 29.28, $\left.\mathrm{df}=26, P=0.30 ; \mathrm{I}^{2}=11 \%\right)$.

For topical application, the use of TXA did not reduce the need for allogeneic blood transfusion (RR 1.02, 95\% CI

\begin{tabular}{|c|c|c|c|c|c|c|c|c|c|c|}
\hline Studv or Subgroup & $\begin{array}{l}\text { TXA } \\
\text { Events }\end{array}$ & Total & $\begin{array}{l}\text { Contr } \\
\text { Events }\end{array}$ & $\begin{array}{l}\text { ol } \\
\text { Total }\end{array}$ & Weight & $\begin{array}{c}\text { Risk Ratio } \\
\text { M-H, Random, } 95 \% \mathrm{Cl}\end{array}$ & & $\begin{array}{r}\text { Risk F } \\
\text { M-H. Rando }\end{array}$ & $\begin{array}{l}\text { Ratio } \\
\text { om. } 95 \% \mathrm{Cl}\end{array}$ & \\
\hline Ahn, S. W., et al. (2012) & 20 & 38 & 27 & 38 & $3.9 \%$ & $0.74[0.51,1.07]$ & & $\rightarrow$ & & \\
\hline Andreasen, J.J. and C. Nielsen (2004) & 5 & 23 & 6 & 23 & $0.7 \%$ & $0.83[0.30,2.35]$ & & & & \\
\hline Armellin, G., et al. (2001) & 35 & 143 & 63 & 140 & $4.2 \%$ & $0.54[0.39,0.77]$ & & $\rightarrow$ & & \\
\hline Baric (2007) & 51 & 97 & 51 & 96 & $5.4 \%$ & $0.99[0.76,1.29]$ & & & - & \\
\hline Baric, D., et al. (2011) & 27 & 40 & 14 & 20 & $4.0 \%$ & $0.96[0.67,1.38]$ & & & - & \\
\hline Bonis (2000) & 3 & 20 & 4 & 20 & $0.4 \%$ & $0.75[0.19,2.93]$ & & & & \\
\hline Brown, R. S., et al. (1997) & 18 & 60 & 20 & 30 & $2.8 \%$ & $0.45[0.28,0.71]$ & & - & & \\
\hline Casati, $V_{.}$, et al. (2001) & 2 & 20 & 4 & 20 & $0.3 \%$ & $0.50[0.10,2.43]$ & & & & \\
\hline Casati, V., et al. (2004) & 9 & 52 & 13 & 50 & $1.3 \%$ & $0.67[0.31,1.42]$ & & & & \\
\hline De Peppo, A. P., et al. (1995) & 1 & 15 & 3 & 15 & $0.2 \%$ & $0.33[0.04,2.85]$ & & & & \\
\hline Diprose, P., et al. (2005) & 20 & 60 & 27 & 60 & $2.9 \%$ & $0.74[0.47,1.17]$ & & $\longrightarrow$ & & \\
\hline Esfandiari, B. R., et al. (2013) & 22 & 75 & 43 & 75 & $3.4 \%$ & $0.51[0.34,0.76]$ & & & & \\
\hline Hardy, J. F., et al. (1998) & 28 & 43 & 29 & 45 & $4.7 \%$ & $1.01[0.74,1.38]$ & & & + & \\
\hline Hashemi, J., et al. (2011) & 15 & 50 & 29 & 50 & $2.6 \%$ & $0.52[0.32,0.84]$ & & & & \\
\hline Jares, M., et al. (2003) & 2 & 22 & 7 & 25 & $0.4 \%$ & $0.32[0.08,1.40]$ & & & & \\
\hline Katsaros, D., et al. (1996) & 11 & 104 & 27 & 106 & $1.7 \%$ & $0.42[0.22,0.79]$ & & - & & \\
\hline Kimenai, D. M., et al. (2016) & 94 & 245 & 94 & 249 & $6.3 \%$ & $1.02[0.81,1.27]$ & & & - & \\
\hline Later, A. F. L., et al(2009) & 57 & 99 & 73 & 103 & $6.6 \%$ & $0.81[0.66,1.00]$ & & $\rightarrow$ & & \\
\hline Mehr-Aein, $A_{\text {, }}$ et al. (2006) & 5 & 33 & 8 & 33 & $0.8 \%$ & $0.63[0.23,1.71]$ & & & & \\
\hline Misfeld, M., et al. (1998) & 2 & 14 & 5 & 14 & $0.4 \%$ & $0.40[0.09,1.73]$ & & & & \\
\hline Murphy, G.J., et al. (2006) & 13 & 50 & 14 & 50 & $1.7 \%$ & $0.93[0.49,1.77]$ & & $\longrightarrow$ & & \\
\hline Myles, P. S., et al. (2017) & 759 & 2311 & 1086 & 2320 & $9.4 \%$ & $0.70[0.65,0.75]$ & & 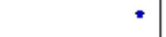 & & \\
\hline Pinosky, M. L., et al. (1997) & 11 & 20 & 9 & 19 & $1.8 \%$ & $1.16[0.63,2.15]$ & & . & & \\
\hline pleym (2003) & 7 & 40 & 8 & 40 & $0.9 \%$ & $0.88[0.35,2.18]$ & & 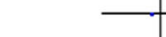 & & \\
\hline Rousou, J. A., et al. (1995) & 72 & 206 & 106 & 209 & $6.2 \%$ & $0.69[0.55,0.87]$ & & $\rightarrow$ & & \\
\hline Shi, J., et al. (2013) & 166 & 274 & 221 & 278 & $8.7 \%$ & $0.76[0.68,0.85]$ & & - & & \\
\hline Shore-Lesserson, L., et al. (1996) & 10 & 17 & 12 & 13 & $3.2 \%$ & $0.64[0.42,0.98]$ & & $\rightarrow-$ & & \\
\hline Speekenbrink, R. G. H., et al. (1995) & 2 & 15 & 4 & 15 & $0.3 \%$ & $0.50[0.11,2.33]$ & & & & \\
\hline Taghaddomi, R. J., et al. (2009) & 8 & 50 & 31 & 50 & $1.6 \%$ & $0.26[0.13,0.50]$ & & & & \\
\hline Wang, G., et al. (2012) & 37 & 116 & 54 & 115 & $4.4 \%$ & $0.68[0.49,0.94]$ & & $\rightarrow$ & & \\
\hline Zabeeda, D., et al. (2002) & 9 & 25 & 25 & 25 & $2.5 \%$ & $0.37[0.22,0.62]$ & & - & & \\
\hline Zabeeda, D., et al. (2002) & 0 & 0 & 0 & 0 & & Not estimable & & & & \\
\hline Zhang, Y., et al. (2018) & 52 & 100 & 73 & 102 & $6.3 \%$ & $0.73[0.58,0.91]$ & & $\rightarrow$ & & \\
\hline Total $(95 \% \mathrm{Cl})$ & & 4477 & & 4448 & $100.0 \%$ & $0.71[0.65,0.78]$ & & 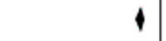 & & \\
\hline Total events & 1573 & & 2190 & & & & & & & \\
\hline \multicolumn{7}{|c|}{$\begin{array}{l}\text { Heterogeneity: } \text { Tau }^{2}=0.02 ; \mathrm{Chi}^{2}=60.98, \mathrm{df}=31(P=0.001) ; \mathrm{I}^{2}=49 \% \\
\text { Test for overall effect: } Z=7.26(P<0.00001)\end{array}$} & 0.01 & $\begin{array}{ll}0.1 & 1 \\
\text { Favours } & \text { [TXA] }\end{array}$ & 1010 & 100 \\
\hline
\end{tabular}




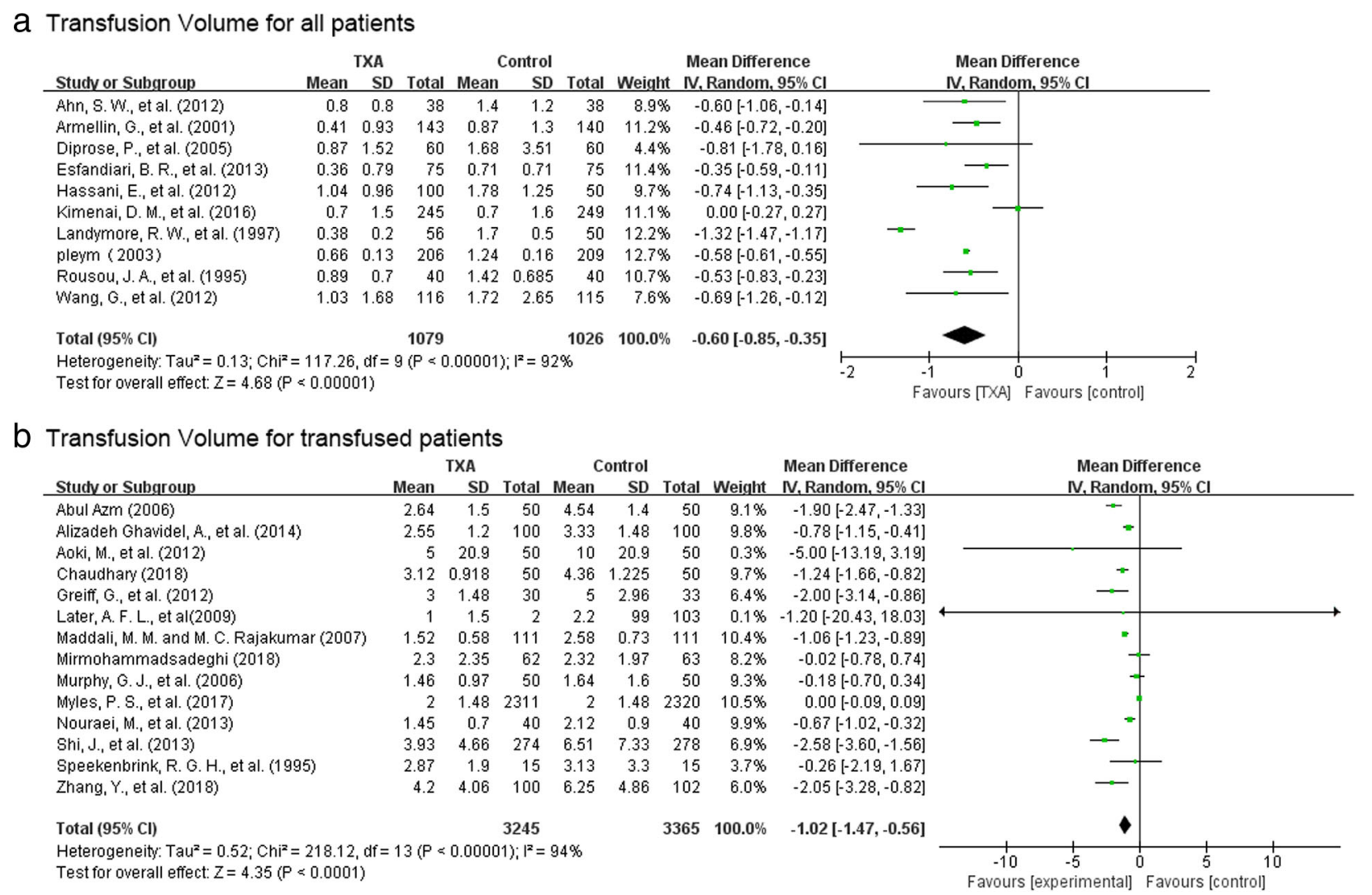

Fig. 5 TXA vs control - transfusion volume; a transfusion volume for all patients, $\mathbf{b}$ transfusion volume for transfused patients

0.87 to $1.20, P=0.76)$. Heterogeneity between these trials was low $\left(\mathrm{Chi}^{2}=2.18, \mathrm{df}=3, \mathrm{P}=0.54 ; \mathrm{I}^{2}=0 \%\right.$ ) (Additional file 11: Figure S8).

\section{Transfusion rate_- bolus and bolus plus continuous infusion}

There were 10 trials on bolus infusion and 18 trials on bolus plus continuous infusion of TXA that reported data for transfusion rate. In the 18 trials with 2310 patients that had bolus plus continuous infusion of TXA, the use of TXA significantly reduce the need for allogeneic blood transfusion by a relative 30\% (RR 0.70, 95\% CI 0.65 to $0.76, \quad \mathrm{P}<0.00001)$. Heterogeneity between these trials was moderate $\left(\mathrm{Chi}^{2}=18.92, \mathrm{df}=17, P=0.33\right.$; $\left.\mathrm{I}^{2}=10 \%\right)$.

In the 10 trials with 5828 patients on bolus injection of TXA, the use of TXA significantly reduce the need for allogeneic blood transfusion by a relative $31 \%$ (RR $0.69,95 \%$ CI 0.64 to $0.74, \mathrm{P}<0.00001)$. Heterogeneity between these trials was moderate $\left(\mathrm{Chi}^{2}=15.05, \mathrm{df}=9\right.$, $P=0.09 ; \mathrm{I}^{2}=40 \%$ ) (Fig. 9).

\section{Transfusion rate-_high and low dose regimen}

For bolus plus continuous infusion, there were 6 trials with low-dose regimen $(\leq 10 \mathrm{mg} / \mathrm{kg}+1 \mathrm{mg} / \mathrm{kg} / \mathrm{h})$ and 12 trials with high-dose regimen. For bolus delivery alone, there were 5 trials with low-dose $\operatorname{regimen}(<50 \mathrm{mg} / \mathrm{kg})$ and 4 trials with high-dose regimen.

For bolus plus continuous infusion, in the 6 lowdosage trials with 265 patients, the use of TXA significantly reduce the need for allogeneic blood transfusion by a relative $50 \%$ (RR $0.50,95 \%$ CI 0.38 to 0.67 , $\mathrm{P}<0.00001)$. Heterogeneity between these trials was moderate $\left(\mathrm{Chi}^{2}=8.94, \mathrm{df}=5, P=0.11 ; \mathrm{I}^{2}=44 \%\right)$. In the 12 high-dosage trials with 2043 patients, the use of TXA significantly reduce the need for allogeneic blood transfusion by a relative $29 \%$ (RR $0.71,95 \%$ CI 0.65 to 0.71 , $P<0.00001)$. Heterogeneity between trials was moderate $\left(\mathrm{Chi}^{2}=13.84, \mathrm{df}=11, P=0.24 ; \mathrm{I}^{2}=21 \%\right)$.

For bolus infusion alone, in the 5 low-dosage trials with 496 patients, the use of TXA significantly reduced the need for allogeneic blood transfusion by a relative $39 \%$ (RR $0.61,95 \%$ CI 0.47 to $0.79, P=0.0001$ ). Heterogeneity between these trials was $\operatorname{low}\left(\mathrm{Chi}^{2}=3.40, \mathrm{df}=4\right.$, $\left.P=0.49 ; \mathrm{I}^{2}=0 \%\right)$.In the 4 high-dosage trials with 5212 patients, the use of TXA significantly reduced the need for allogeneic blood transfusion by $31 \%$ (RR 0.69, 95\% CI 0.65 to $0.74, \mathrm{P}<0.00001)$. Heterogeneity between these trials was significant $\left(\mathrm{Chi}^{2}=10.17, \mathrm{df}=3, P=0.02\right.$; $\mathrm{I}^{2}=71 \%$ ) (Fig. 10). 


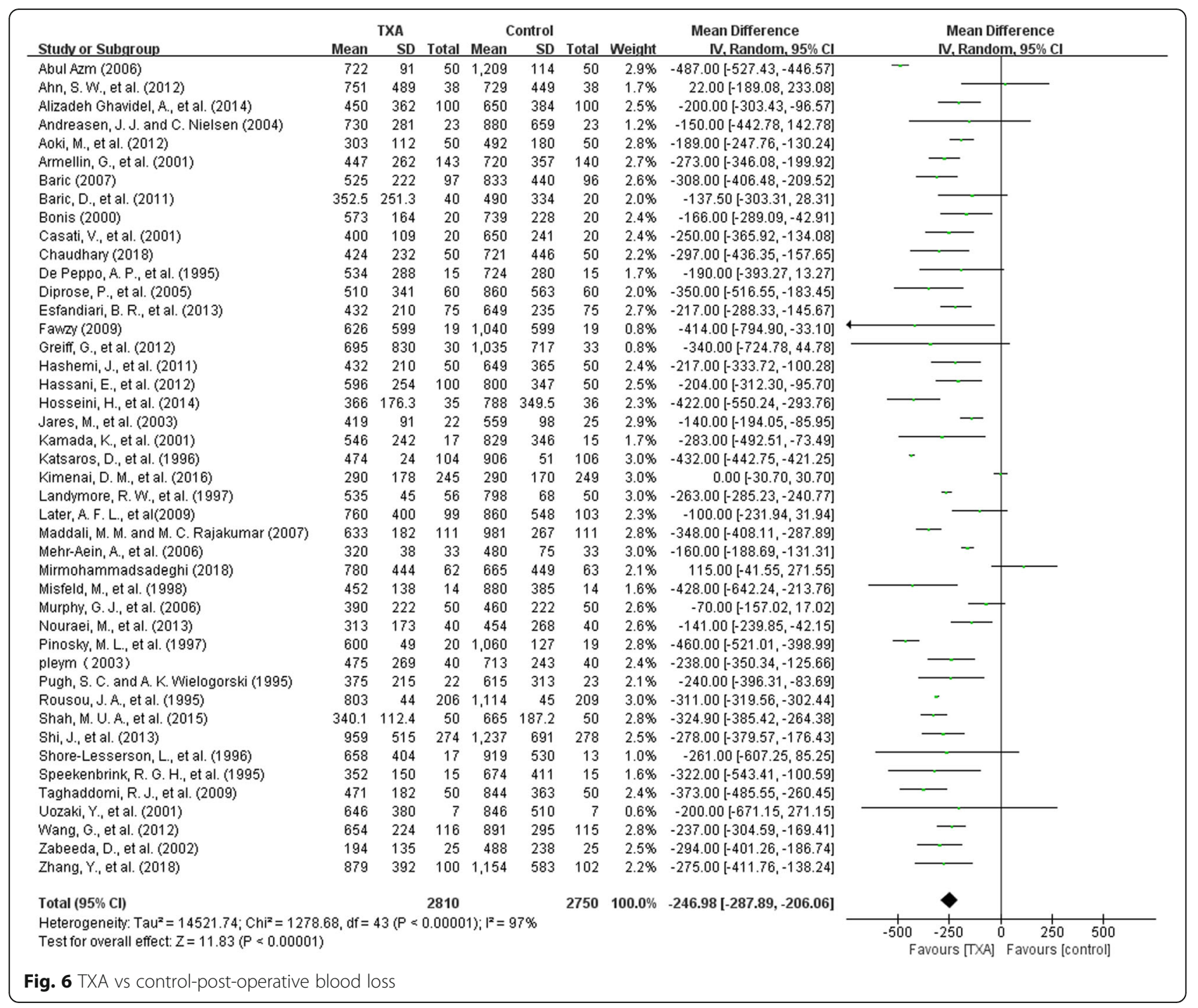

\section{Seizure-high and low dose regimen}

Among the 49 studies included, only 8 trials provided data on seizure attack. Since there were too few studies on seizure, we combined bolus with bolus plus continuous infusion together when analyzing high and low dose regimen $(<50$ $\mathrm{mg} / \mathrm{kg}$ or $\leq 10 \mathrm{mg} / \mathrm{kg}+1 \mathrm{mg} / \mathrm{kg} / \mathrm{h}$ ). Five trials on high dose regimen and 3 trials on low dose regimen were analyzed.

In the 5 high-dose trials with 5807 patients, the use of high-dosage TXA significantly increased the risk of seizure attack by 4.83 times (RR 4.83, 95\% CI 1.75 to $13.33, P=$ 0.002). Heterogeneity between these trials was low $\left(\mathrm{Chi}^{2}=\right.$ 1.37, $\left.\mathrm{df}=2, P=0.50 ; \mathrm{I}^{2}=0 \%\right)$. In the 3 low-dosage trials with 313 patients, no seizure occurred in any of the trials, and the impact of low-dosage TXA on seizure attack could not be assessed (Fig. 11).

\section{Impact of trial quality}

We did a subgroup analysis on transfusion rate for studies with low and unclear risk of bias only and found that the use of TXA reduced the rate of allogeneic blood transfusion by a relative $29 \%$ (RR $0.71,95 \%$ CI 0.68 to $0.75, \mathrm{P}<0.00001)$. Heterogeneity between these trials was significant $\left(\mathrm{Chi}^{2}=59.50, \mathrm{df}=27, P=0.0003 ; \mathrm{I}^{2}=55 \%\right)$ (Additional file 12: Figure S9). This result was largely the same with results including all studies. Excluding trials with high risk of bias didn't make a difference.

\section{Publication bias}

Funnel plot comparing TXA with control group concerning transfusion rate was provided in Additional file 13: Figure S10. No obvious asymmetry was seen from the funnel plot, and thus no publication bias was detected.

\section{Discussion}

In this meta-analysis, we strengthened the results that TXA significantly reduced blood loss, transfusion requirement and re-operation rate in adult cardiac surgery. In addition to updating previous findings, we tried to 


\begin{tabular}{|c|c|c|c|c|c|c|c|c|c|c|}
\hline Study or Subgroup & $\begin{array}{r}\text { TXA } \\
\text { Events }\end{array}$ & Total & \multicolumn{2}{|c|}{ Control } & Weight & $\begin{array}{c}\text { Risk Ratio } \\
\text { M-H, Fixed, 95\% Cl }\end{array}$ & \multicolumn{4}{|c|}{$\begin{array}{c}\text { Risk Ratio } \\
\text { M-H, Fixed, 95\% Cl }\end{array}$} \\
\hline Alizadeh Ghavidel, A., et al. (2014) & 5 & 100 & 5 & 100 & $2.9 \%$ & $1.00[0.30,3.35]$ & & & & \\
\hline Andreasen, J.J. and C. Nielsen (2004) & 1 & 23 & 6 & 23 & $3.4 \%$ & $0.17[0.02,1.28]$ & & & & \\
\hline Aoki, M., et al. (2012) & 0 & 50 & 0 & 50 & & Not estimable & & & & \\
\hline Armellin, G., et al. (2001) & 4 & 143 & 5 & 140 & $2.9 \%$ & $0.78[0.21,2.86]$ & & & & \\
\hline Brown, R. S., et al. (1997) & 0 & 60 & 0 & 30 & & Not estimable & & & & \\
\hline Casati, $Y$., et al. (2001) & 0 & 20 & 0 & 20 & & Not estimable & & & & \\
\hline Casati, V., et al. (2004) & 1 & 52 & 3 & 50 & $1.7 \%$ & $0.32[0.03,2.98]$ & & & & \\
\hline De Peppo, A. P., et al. (1995) & 1 & 15 & 0 & 15 & $0.3 \%$ & $3.00[0.13,68.26]$ & & & & \\
\hline Diprose, P., et al. (2005) & 5 & 60 & $?$ & 60 & $4.0 \%$ & $0.71[0.24,2.13]$ & & & — & \\
\hline Esfandiari, B. R., et al. (2013) & 1 & 75 & 1 & 75 & $0.6 \%$ & $1.00[0.06,15.69]$ & & & & \\
\hline Greiff, G., et al. (2012) & 6 & 30 & 9 & 33 & $4.9 \%$ & $0.73[0.30,1.82]$ & & & 一 & \\
\hline Hardy, J. F., et al. (1998) & 1 & 43 & 3 & 45 & $1.7 \%$ & $0.35[0.04,3.23]$ & & & & \\
\hline Hashemi, J., et al. (2011) & 1 & 50 & 1 & 50 & $0.6 \%$ & $1.00[0.06,15.55]$ & & & & \\
\hline Hosseini, H., et al. (2014) & 0 & 35 & 0 & 36 & & Not estimable & & & & \\
\hline Jares, M., et al. (2003) & 0 & 25 & 1 & 25 & $0.9 \%$ & $0.33[0.01,7.81]$ & & & & \\
\hline Katsaros, D., et al. (1996) & 1 & 104 & 5 & 106 & $2.8 \%$ & $0.20[0.02,1.72]$ & & & - & \\
\hline Kimenai, D. M., et al. (2016) & 12 & 245 & 10 & 249 & $5.7 \%$ & $1.22[0.54,2.77]$ & & - & & \\
\hline Later, A. F. L., et al(2009) & 14 & 99 & 14 & 103 & $7.8 \%$ & $1.04[0.52,2.07]$ & & - & - & \\
\hline Maddali, M. M. and M. C. Rajakumar (2007) & 3 & 111 & 3 & 111 & $1.7 \%$ & $1.00[0.21,4.85]$ & & & & \\
\hline Mehr-Aein, A., et al. (2006) & 0 & 33 & 1 & 33 & $0.9 \%$ & $0.33[0.01,7.90]$ & & & & \\
\hline Murphy, G. J., et al. (2006) & 1 & 50 & 0 & 50 & $0.3 \%$ & $3.00[0.13,71.92]$ & & & & \\
\hline Myles, P. S., et al. (2017) & 32 & 2311 & 65 & 2320 & $37.0 \%$ & $0.49[0.32,0.75]$ & & $\rightarrow-$ & & \\
\hline Nouraei, M., et al. (2013) & 0 & 40 & 0 & 40 & & Not estimable & & & & \\
\hline pleym (2003) & 0 & 40 & 1 & 40 & $0.9 \%$ & $0.33[0.01,7.95]$ & & & & \\
\hline Pugh, S. C. and A. K. Wielogorski (1995) & 0 & 23 & 2 & 23 & $1.4 \%$ & $0.20[0.01,3.95]$ & & 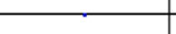 & & \\
\hline Rousou, J. A., et al. (1995) & 10 & 206 & 9 & 209 & $5.1 \%$ & $1.13[0.47,2.72]$ & & & & \\
\hline Shi, J., et al. (2013) & 5 & 274 & 19 & 279 & $10.7 \%$ & $0.27[0.10,0.71]$ & & & & \\
\hline Shore-Lesserson, L., et al. (1996) & 0 & 17 & 0 & 13 & & Not estimable & & & & \\
\hline Speekenbrink, R. G. H., et al. (1995) & 0 & 15 & 0 & 15 & & Not estimable & & & & \\
\hline Uozaki, Y., et al. (2001) & 1 & 7 & 1 & 7 & $0.6 \%$ & $1.00[0.08,13.02]$ & & & & \\
\hline Wang, G., et al. (2012) & 0 & 116 & 2 & 115 & $1.4 \%$ & $0.20[0.01,4.09]$ & & & & \\
\hline Zhang, Y., et al. (2018) & 0 & 0 & 0 & 0 & & Not estimable & & & & \\
\hline Total $(95 \% \mathrm{Cl})$ & & 4472 & & 4465 & $100.0 \%$ & $0.62[0.49,0.79]$ & & $\bullet$ & & \\
\hline Total events & 105 & & 173 & & & & & & & \\
\hline $\begin{array}{l}\text { Heterogeneity: } \mathrm{Chi}^{2}=18.86, \mathrm{df}=23(\mathrm{P}=0.7 \\
\text { Test for overall effect: } Z=3.99(P<0.0001)\end{array}$ & $; 1^{2}=0 \%$ & & & & & & 0.01 & $\begin{array}{c}0.1 \\
\text { Favours [TX] }\end{array}$ & $\begin{array}{cc}1 & 10 \\
\text { Favours [control] }\end{array}$ & 100 \\
\hline
\end{tabular}

\begin{tabular}{|c|c|c|c|c|c|c|c|c|c|c|}
\hline Studv or Subgroup & $\begin{array}{l}\text { TXA } \\
\text { Events } \\
\end{array}$ & Total & $\begin{array}{l}\text { Contro } \\
\text { Events }\end{array}$ & Iotal & Weight & $\begin{array}{c}\text { Risk Ratio } \\
\text { M-H, Random, } 95 \% \mathrm{Cl}\end{array}$ & \multicolumn{4}{|c|}{$\begin{array}{c}\text { Risk Ratio } \\
\text { M-H, Random, } 95 \% \mathrm{Cl}\end{array}$} \\
\hline Aoki, M., et al. (2012) & 0 & 50 & 0 & 50 & & Not estimable & & & & \\
\hline Esfandiari, B. R., et al. (2013) & 0 & 75 & 0 & 75 & & Not estimable & & & & \\
\hline Greiff, G., et al. (2012) & 0 & 30 & 0 & 33 & & Not estimable & & & & \\
\hline Hashemi, J., et al. (2011) & 0 & 50 & 0 & 50 & & Not estimable & & & & \\
\hline Katsaros, D., et al. (1996) & 0 & 104 & 0 & 106 & & Not estimable & & & & \\
\hline Kimenai, D. M., et al. (2016) & 0 & 245 & 1 & 249 & $11.4 \%$ & $0.34[0.01,8.28]$ & & & & \\
\hline Later, A. F. L., et al(2009) & 0 & 99 & 0 & 103 & & Not estimable & & & & \\
\hline Myles, P. S., et al. (2017) & 15 & 2311 & 2 & 2320 & $41.8 \%$ & $7.53[1.72,32.89]$ & & & 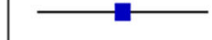 & \\
\hline Nouraei, M., et al. (2013) & 0 & 40 & 0 & 40 & & Not estimable & & & & \\
\hline Shi, J., et al. (2013) & 4 & 274 & 2 & 278 & $34.1 \%$ & $2.03[0.37,10.99]$ & & & & \\
\hline Zhang, Y., et al. (2018) & 2 & 100 & 0 & 102 & $12.7 \%$ & $5.10[0.25,104.90]$ & & & & \\
\hline Total (95\% Cl) & & 3378 & & 3406 & $100.0 \%$ & $3.21[1.04,9.90]$ & & & & \\
\hline Total events & 21 & & 5 & & & & & & & \\
\hline $\begin{array}{l}\text { Heterogeneity: } \mathrm{Tau}^{2}=0.22 ; \mathrm{Ch} \\
\text { Test for overall effect: } Z=2.03\end{array}$ & $\begin{array}{l}=3.57, d f \\
P=0.04)\end{array}$ & $f=3(P$ & $=0.31) ;$ & ${ }^{2}=16^{\circ}$ & & & 0.01 & $\begin{array}{l}0.1 \\
\text { Favours [XA] }\end{array}$ & $\begin{array}{c}10 \\
\text { Favours [control] }\end{array}$ & 100 \\
\hline
\end{tabular}


Table 1 TXA vs Control for mortality and thrombotic events

\begin{tabular}{llllll}
\hline Endpoints & Number of studies/reference & Overall participants & Effect estimate (RR, 95\% CI) & P & Heterogeneity $\left(1^{2}\right)$ \\
\hline Mortality & 29 & 8907 & $0.78(0.54,1.14)$ & 0.20 & $0 \%$ \\
Stroke & 32 & 9257 & $0.88(0.61,1.28)$ & 0.50 & $0 \%$ \\
Myocardial infarction & 32 & 8688 & $0.89(0.77,1.04)$ & 0.67 & $0 \%$ \\
Pulmonary embolism & 18 & 6587 & $1.08(0.59,2.00)$ & 0.60 & $0 \%$ \\
Renal dysfunction & 19 & 7210 & $0.99(0.77,1.27)$ & 0.92 & $0 \%$ \\
\hline
\end{tabular}

answer three important questions: whether TXA is effective for all kinds of elective heart surgeries on adults, whether TXA leads to adverse effects, what's the preferable dose regimen and delivery method for TXA.

We found TXA to be effective for both off-pump and on-pump operations. Although off-pump patients didn't undergo $\mathrm{CPB}$, they were inevitability exposed be heparin, protamine and even greater surgical trauma than onpump patients, and we found that these patients could benefit as much as, if not more than on-pump patient from TXA.

TXA did not show any trend to increase the risk of thrombotic events including myocardial infarction, stroke and pulmonary embolism, and wasn't associated

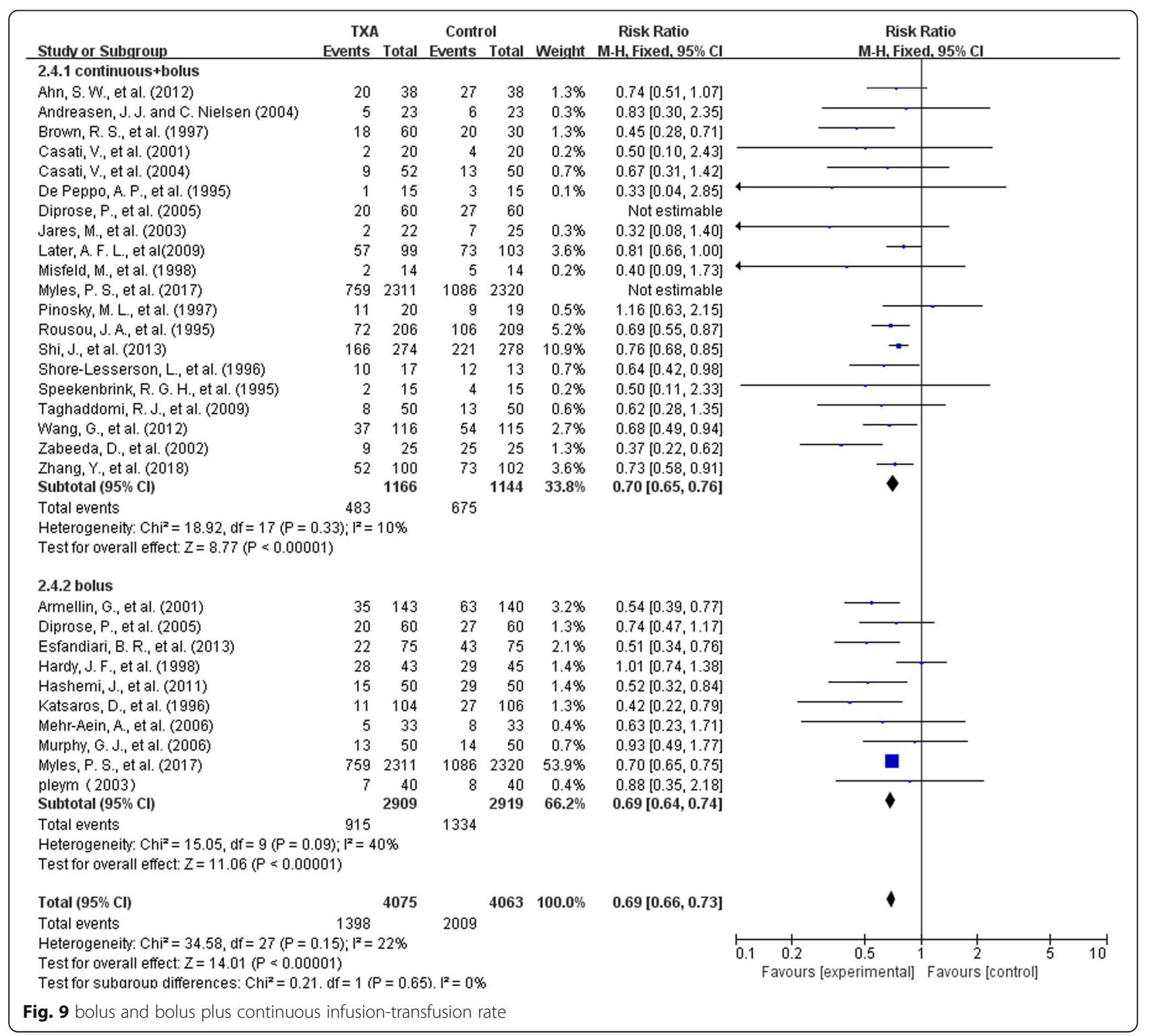




\begin{tabular}{|c|c|c|c|c|c|c|c|c|c|c|c|}
\hline \multirow{2}{*}{$\begin{array}{l}\text { Studv or Subgroup } \\
\text { 2.5.1 } \mathrm{B}+\mathrm{C} \text { (low dose) }\end{array}$} & $\begin{array}{l}\text { TXA } \\
\text { Events }\end{array}$ & Total & \multicolumn{2}{|c|}{$\begin{array}{l}\text { Control } \\
\text { Events Total }\end{array}$} & Weight & \multirow[t]{2}{*}{$\begin{array}{l}\text { Risk Ratio } \\
\text { M-H, Fixed, } 95 \% \mathrm{Cl}\end{array}$} & \multicolumn{5}{|c|}{$\begin{array}{l}\text { Risk Ratio } \\
\text { M-H, Fixed, } 95 \% \mathrm{Cl}\end{array}$} \\
\hline & & & & & & & & & & & \\
\hline Brown, R. S., et al. (1997) & 18 & 60 & 20 & 30 & $1.3 \%$ & $0.45[0.28,0.71]$ & & & & & \\
\hline De Peppo, A. P., et al. (1995) & 1 & 15 & 3 & 15 & $0.1 \%$ & $0.33[0.04,2.85]$ & & & & & \\
\hline Misfeld, M., et al. (1998) & 2 & 14 & 5 & 14 & $0.2 \%$ & $0.40[0.09,1.73]$ & & & & & \\
\hline Pinosky, M. L., et al. (1997) & 11 & 20 & 9 & 19 & $0.5 \%$ & $1.16[0.63,2.15]$ & & & & & \\
\hline Share-Lesserson, L., et al. (1996) & 10 & 17 & 12 & 13 & & Not estimable & & & & & \\
\hline Speekenbrink, R. G. H., et al. (1995) & 2 & 14 & 5 & 14 & $0.2 \%$ & $0.40[0.09,1.73]$ & & & & & \\
\hline Zabeeda, D., et al. (2002) & 9 & 25 & 25 & 25 & $1.3 \%$ & $0.37[0.22,0.62]$ & & & & & \\
\hline Subtotal $(95 \% \mathrm{Cl})$ & & 148 & & 117 & $3.7 \%$ & $0.50[0.38,0.67]$ & & & & & \\
\hline Total events & 43 & & 67 & & & & & & & & \\
\hline \multicolumn{12}{|c|}{$\begin{array}{l}\text { Heterogeneity: } \mathrm{Chi}^{2}=8.94, \mathrm{df}=5(\mathrm{P}=0.11) ; \mathrm{l}^{2}=44 \% \\
\text { Test for overall effect } Z=476(\mathrm{P}<0.00001)\end{array}$} \\
\hline \multicolumn{12}{|l|}{ 2.5.2 $\mathrm{B}+\mathrm{C}$ (high dose) } \\
\hline Ahn, S. W., et al. (2012) & 20 & 38 & 27 & 38 & $1.3 \%$ & $0.74[0.51,1.07]$ & & & & & \\
\hline Andreasen, J.J. and C. Nielsen (2004) & 5 & 23 & 6 & 23 & $0.3 \%$ & $0.83[0.30,2.35]$ & & & & & \\
\hline Casati, V., et al. (2001) & 2 & 20 & 4 & 20 & $0.2 \%$ & $0.50[0.10,2.43]$ & & & & & \\
\hline Casati, V., et al. (2004) & 9 & 52 & 13 & 50 & $0.7 \%$ & $0.67[0.31,1.42]$ & & & & & \\
\hline Jares, M., et al. (2003) & 2 & 22 & 7 & 25 & $0.3 \%$ & $0.32[0.08,1.40]$ & & & & & \\
\hline Later, A. F. L., et al(2009) & 57 & 99 & 73 & 103 & $3.6 \%$ & $0.81[0.66,1.00]$ & & $\rightarrow$ & & & \\
\hline Rousou, J. A., et al. (1995) & 72 & 206 & 106 & 209 & $5.3 \%$ & $0.69[0.55,0.87]$ & & $\rightarrow$ & & & \\
\hline Shi, J., et al. (2013) & 166 & 274 & 221 & 278 & $11.0 \%$ & $0.76[0.68,0.85]$ & & $=$ & & & \\
\hline Shore-Lesserson, L., et al. (1996) & 10 & 17 & 12 & 13 & $0.7 \%$ & $0.64[0.42,0.98]$ & & & & & \\
\hline Taghaddomi, R. J., et al. (2009) & 8 & 50 & 31 & 50 & $1.5 \%$ & $0.26[0.13,0.50]$ & & & & & \\
\hline Wang, G., et al. (2012) & 37 & 116 & 54 & 115 & $2.7 \%$ & $0.68[0.49,0.94]$ & & & & & \\
\hline Zhang, Y., et al. (2018) & 52 & 100 & 73 & 102 & $3.6 \%$ & $0.73[0.58,0.91]$ & & & & & \\
\hline Subtotal $(95 \% \mathrm{Cl})$ & & 1017 & & 1026 & $31.2 \%$ & $0.71[0.65,0.77]$ & & $\bullet$ & & & \\
\hline Total events & 440 & & 627 & & & & & & & & \\
\hline \multicolumn{12}{|c|}{ Heterogeneity: $\mathrm{Chi}^{2}=13.84, \mathrm{df}=11(\mathrm{P}=0.24) ; \mathrm{I}^{2}=21 \%$} \\
\hline \multicolumn{12}{|c|}{ Test for overall effect: $Z=8.35(P<0.00001)$} \\
\hline \multicolumn{12}{|l|}{ 2.5.3 B (low dose) } \\
\hline Esfandiari, B. R., et al. (2013) & 22 & 75 & 43 & 75 & $2.1 \%$ & $0.51[0.34,0.76]$ & & & & & \\
\hline Hashemi, J., et al. (2011) & 15 & 50 & 29 & 50 & $1.4 \%$ & $0.52[0.32,0.84]$ & & & & & \\
\hline Mehr-Aein, $A_{\text {., et al }}(2006)$ & 5 & 33 & 8 & 33 & $0.4 \%$ & $0.63[0.23,1.71]$ & & & & & \\
\hline Murphy, G. J., et al. (2006) & 13 & 50 & 14 & 50 & $0.7 \%$ & $0.93[0.49,1.77]$ & & & & & \\
\hline pleym (2003) & 7 & 40 & 8 & 40 & $0.4 \%$ & $0.88[0.35,2.18]$ & & & & & \\
\hline Subtotal $(95 \% \mathrm{Cl})$ & & 248 & & 248 & $5.1 \%$ & $0.61[0.47,0.79]$ & & & & & \\
\hline Total events & 62 & & 102 & & & & & & & & \\
\hline \multicolumn{12}{|c|}{ Heterogeneity: $\mathrm{Chi}^{2}=3.40, \mathrm{df}=4(\mathrm{P}=0.49) ; \mathrm{I}^{2}=0 \%$} \\
\hline \multicolumn{12}{|c|}{ Test for overall effect: $Z=3.79(P=0.0001)$} \\
\hline \multicolumn{12}{|l|}{ 2.5.4 B (high dose) } \\
\hline Armellin, G., et al. (2001) & 35 & 143 & 63 & 140 & $3.2 \%$ & $0.54[0.39,0.77]$ & & - & & & \\
\hline Hardy, J. F., et al. (1998) & 28 & 43 & 29 & 45 & $1.4 \%$ & $1.01[0.74,1.38]$ & & & & & \\
\hline Katsaros, D., et al. (1996) & 11 & 104 & 27 & 106 & $1.3 \%$ & $0.42[0.22,0.79]$ & & & & & \\
\hline Myles, P. S., et al. (2017) & 759 & 2311 & 1086 & 2320 & $54.1 \%$ & $0.70[0.65,0.75]$ & & [ & & & \\
\hline Subtotal $(95 \% \mathrm{Cl})$ & & 2601 & & 2611 & $60.0 \%$ & $0.69[0.65,0.74]$ & & 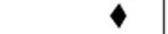 & & & \\
\hline Total events & 833 & & 1205 & & & & & & & & \\
\hline \multirow{2}{*}{\multicolumn{12}{|c|}{$\begin{array}{l}\text { Heterogeneity: } \mathrm{Chi}^{2}=10.17, \mathrm{df}=3(\mathrm{P}=0.02) ; \mathrm{I}^{2}=71 \% \\
\text { Test for overall effect: } Z=10.34(P \approx 0.00001)\end{array}$}} \\
\hline & & & & & & & & & & & \\
\hline Total $(95 \% \mathrm{Cl})$ & & 4014 & & 4002 & $100.0 \%$ & $0.69[0.65,0.72]$ & & 1 & & & \\
\hline Total events & 1378 & & 2001 & & & & & & & & \\
\hline Heterogeneity: $\mathrm{Chi}^{2}=43.76, \mathrm{df}=26(\mathrm{P}=$ & $.02) ; 1^{2}=4$ & $41 \%$ & & & & & $\begin{array}{ll} \\
0.1 & 0.2\end{array}$ & & 12 & 10 & \\
\hline $\begin{array}{l}\text { Test for overall effect: } Z=14.34 \text { ( } P<0.0 \\
\text { Test for subaroun differences: } \mathrm{Chi}^{2}=6 .\end{array}$ & 2. $d f=3(P$ & $=0.10$ & $I^{2}=51$ & & & & $\begin{array}{l}0.1 \quad 0.2 \\
\text { Favours }\end{array}$ & $s$ [experimental] & ${ }^{1}$ Favours [control] ${ }^{5}$ & 10 & \\
\hline Fig. 10 high and low dose regimen-t & ansfusion & rate & & & & & & & & & \\
\hline
\end{tabular}

with renal injury. But TXA significantly increased the risk of seizure, which was a result well worth attention. According to our findings, seizure was a rare event in patients underwent cardiac surgeries. There were 11 studies that reported data on epileptic attack and 7 of them reported no attack in both TXA and control groups. In the other 4 studies, seizure happened at a rate of $0.20-1.08 \%$. Given the rare nature of seizure attack, we consider it valuable to look at results from retrospective observational studies. In an observational study of 12,195 patients underwent cardiac surgery, none of the 886 patients in the control group without TXA administration had seizure and 80 out of 9642 patients $(0.83 \%)$ with TXA delivery had seizure [60]. Takagai et al. did a meta-analysis on all kinds of researches concerning the association between TXA and seizure in cardiac surgeries [61]. They identified 16 studies with 45,235 patients, and demonstrated that TXA 


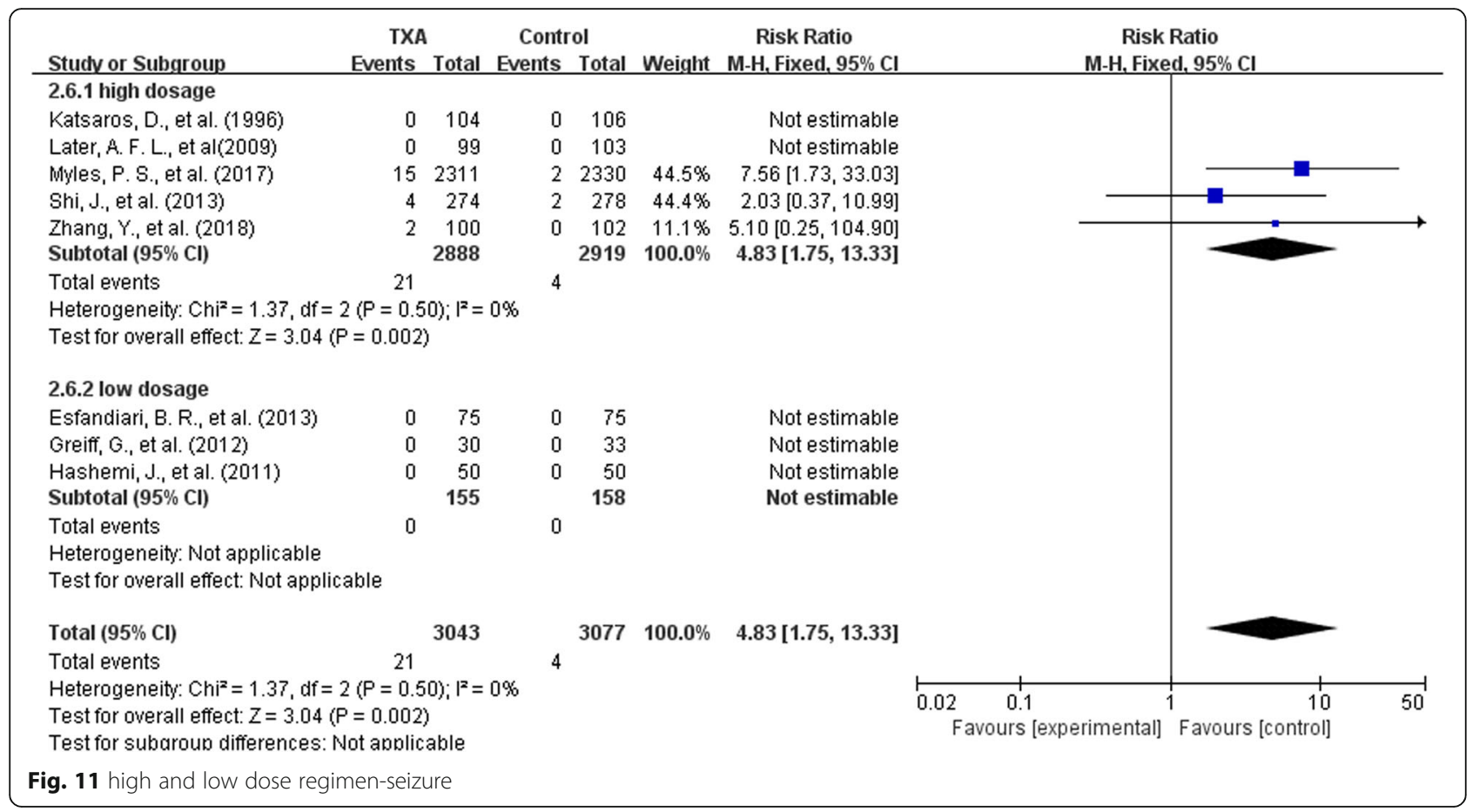

therapy was associated with a statistically significant increase in seizures incidence $(\mathrm{OR}=4.13 ; 95 \% \mathrm{CI}: 2.59$ to 6.57; $P<0.00001)$. A subgroup analysis indicated a significant increase seizure in all subgroups of 5 RCTs, 5 adjusted observational studies, and 6 unadjusted observational studies with no statistically significant subgroup difference $\left(P=0.36 ; \mathrm{I}^{2}=1.5 \%\right)$.

We found that intravenous delivery of TXA significantly reduced transfusion rate by $30 \%$, while topical application did not show any signs to reduce transfusion requirement. This result can be further confirmed by several studies comparing intravenous plus topical use of TXA with intravenous application alone. In a RCT conducted by Taksaudom et al. [62], $1 \mathrm{~g}$ of topical TXA was poured into the pericardial cavity in addition to $1 \mathrm{mg} /$ $\mathrm{kg} / \mathrm{h}$ of intravenous infusion in the experiment group, and only $1 \mathrm{mg} / \mathrm{kg} / \mathrm{h}$ of intravenous infusion was applied in the control group. No significant reduction was seen in post-operative blood loss, and transfusion rate was even higher in the experiment group (75.6\% vs $70.6 \%)$. The same happened for another RCT conducted by Spegar et al. in which $2.5 \mathrm{~g}$ topical TXA was applied in addition to $1 \mathrm{~g}+400 \mathrm{mg} / \mathrm{h}$ of intravenous infusion [63]. According to existing evidence, topical TXA was not effective enough to reduce bleeding and transfusion requirements no matter used alone or combined with intravenous application, and further research is needed to justify its use in cardiac procedures.

Both continuous plus bolus and bolus delivery alone significantly reduced transfusion rate compared to the control group, and bolus plus continuous delivery (RR 0.70) did not seem to be more effective than bolus injection (RR 0.69). In a RCT conducted by Imtiaz, et al., 137 CABG patients were randomly assigned to receive either bolus injection or continuous infusion of $30 \mathrm{mg} / \mathrm{kg}$ TXA, and no difference were found in blood loss or transfusion requirements [64]. This result was in accordance with our finding that bolus and bolus plus continuous delivery were equally effective. We infer that both delivery methods are able to keep the effect compartment concentration above minimum effective concentration during surgery as long as dosage is enough, but peak concentration may be higher if bolus injection alone is applied which may cause more adverse events. However, this hypothesis needs to be tested by pharmacokinetics studies.

From subgroup analysis, we can conclude that low-dose intravenous TXA, no matter delivered as bolus injection or bolus plus continuous infusion, was effective in reducing transfusion requirements. When comparing with high-dose regimen, lose-dose TXA was at least equally effective, and may be even more effective in reducing transfusion rate. The risk ratio for transfusion rate were 0.50 and 0.61 for low-dose bolus and bolus plus continuous infusion separately, while for high-dose regimen risk ratio was 0.71 and 0.69 separately. As we mentioned above, the use of TXA was significantly associated with an increase in seizure attack. In subgroup analysis, TXA increased the risk of seizure only in patients using high-dose regimen, and none of the patients using low-dose TXA 
had seizure attack. These evidences give us the basic consumption that low-dose TXA is enough in reducing transfusion requirement and is less likely to cause seizure.

There were several studies that directly compared the effectiveness of high and low-dose regimen of TXA, but the definition of high and low-dose regimen varied significantly among these studies, we thus consider it improper to combine the results together in meta-analysis. Here we did a qualitative other than quantitative analysis of these studies and see if they were in accordance with our basic consumption.

We identified 6 RCTs that compare transfusion rate between high and low dose regimen [65-72], and none of them showed significant decrease in transfusion rate in the high dose group. Fewer studies reported data on transfusion volume, again no significant decrease were found in allogeneic $\mathrm{RBC}$ transfusion in the high dose group [66, 70]. These results agreed with our previous findings. In terms of postoperative blood loss, the results were less unanimous. We found 8 RCTs comparing blood loss between high and low dose regimen [65-72], among which 5 studies reported no difference in blood loss $[65,66,69$, 71,72 ]. Karski et al. [68] randomly assigned patients to receive 50,100 or $150 \mathrm{mg} / \mathrm{kg}$ of TXA infusion and found that blood loss was significantly higher in the $50 \mathrm{mg} / \mathrm{kg}$ group. Jiménez et al. [67] reported blood loss to be significantly reduced in patients who received $80 \mathrm{mg} / \mathrm{kg}$ TXA compared with patients who received $40 \mathrm{mg} / \mathrm{kg}$ TXA. Sigaut et al. (71)found that blood loss was significantly reduced when $30 \mathrm{mg} / \mathrm{kg}+16 \mathrm{mg} / \mathrm{kg} / \mathrm{h}$ of TXA was infused compared with $10 \mathrm{mg} / \mathrm{kg}+1 \mathrm{mg} / \mathrm{kg} / \mathrm{h}$ of TXA. We identified 3 studies that reported data on seizure $[66,67,70]$, and none of them found significant difference between the high and low-dose group, but there was a trend towards more seizure attack. Jiménez et al. [67] reported seizure rate to be $2 / 80$ in the high-dose group and $0 / 80$ in the low dose group. Sigaut et al. [70] found that seizure happened in 3 out of 285 patients in the high dose group and 1 out of 284 patients in the low dose group. In the study conducted by $\mathrm{Du}$ et al. [66], seizure rate were $1 / 87$ and $1 / 88$ in high and low dose group separately, and the dose regimen used in their study were $30 \mathrm{mg} / \mathrm{kg}+16 \mathrm{mg} / \mathrm{kg} / \mathrm{h}$ for high-dose and $10 \mathrm{mg} / \mathrm{kg}+2 \mathrm{mg} / \mathrm{kg} / \mathrm{h}$ for low-dose regimen. From these trials which directly compared high and low dose TXA, we can conclude that high dose TXA has little effect in reducing transfusion requirement and tends to cause more seizure attacks, but it may be more effective in reducing blood loss compared to low dose regimen.

Basic researches may explain this different dosage requirements. As we've mentioned in the background, TA concentrations required to suppress fibrinolysis and plasmin-induced platelet activation are 10 and $16 \mu \mathrm{g} / \mathrm{ml}$, respectively [7, 8]. TXA concentration of $10 \mu \mathrm{g} / \mathrm{ml}$ will result in $80 \%$ inhibition of tissue plasminogen activator, but in order to achieve 100\% inhibition of tissue plasminogen activator, a tranexamic acid concentration of $100 \mu \mathrm{g} /$ $\mathrm{ml}$ is necessary [73]. Another potential mechanism of TXA action is the increase in thrombin formation, which requires concentrations more than $126 \mu \mathrm{g} / \mathrm{ml}$ to be therapeutic $[10,11]$. It is likely that low-dose TXA as $10 \mathrm{mg} / \mathrm{kg}$ bolus injection followed by $1 \mathrm{mg} / \mathrm{kg} / \mathrm{h}$ continuous infusion is able to achieve a large proportion of TXA efficacy, and the effect added by high dose regimen isn't large enough to further decrease transfusion requirements but may reduce blood loss to certain extent.

We noticed that the stratification for risk of bleeding/ transfusion may be useful in guiding the dosage of TXA. In the RCT conducted by Sigaut et al. [70], no difference in transfusion requirement was found between high and low dose TXA group, but when subgroup analysis was conducted for patients with high risk of transfusion, a significant decrease in transfusion volume were seen in the high-dose group. In this study, the definition of high-risk of transfusion was as follow: "Patients were considered at high risk for transfusion if they were receiving a dual anti-platelet at any time within 5 days of surgery, or in the following cases: repeat coronary artery bypass graft, repeat valve surgery (replacement or repair), combined coronary artery bypass graft and valve surgery, multiple valve surgery, surgery of the aorta, intracardiac tumor ablation, and surgery for endocarditis. All other cardiac surgery procedures were considered low risk." We believe that further researches are needed for patients with high-risk of bleeding to decide whether the dosage of TXA should be raised.

Heterogeneity in transfusion rate existed between trials $\left(\mathrm{I}^{2}=49 \%\right)$. From subgroup analysis, we can see that heterogeneity mainly came from intravenous and topical delivery method (intravenous $\mathrm{I}^{2}=11 \%$, topical $\mathrm{I}^{2}=0 \%$, subgroup differences $\mathrm{P}<0.00001$ ).

There was significant heterogeneity in transfusion volume (transfusion volume for all patients $\mathrm{I}^{2}=92 \%$, transfusion volume for transfused patients $\mathrm{I}^{2}=94 \%$ ) and postoperative blood $\operatorname{loss}\left(\mathrm{I}^{2}=97 \%\right)$. Topical and intravenous delivery explained part of the problem, but we believe that heterogeneity mainly came from two reasons. For transfusion volume, different studies varied in measurement of blood, some used $\mathrm{ml}$ and some used blood unit which was different depending on different countries. For blood loss, studies varied in the time point in measuring postoperative blood loss, form $4 \mathrm{~h}$ to the several days. These sources of heterogeneity were hard to solve by subgroup analysis, and we thus used the random-effect model to estimate the average effect of TXA delivery on these outcomes.

No heterogeneity existed concerning adverse events including reoperation rate, mortality, stroke, myocardial infarction, pulmonary embolism and renal dysfunction. Mild heterogeneity existed concerning seizure $\left(\mathrm{I}^{2}=16 \%\right)$, 
and we've identified from subgroup analysis that this heterogeneity came from different dose regimen (high-dose group $\mathrm{I}^{2}=0 \%$ ).

Sensitivity analysis showed that excluding trials with high risk of bias didn't make a difference on the main result of transfusion rate. While one thing worth noticing was that the RCT conducted by Myles et al. [6] which had the largest sample size among included studies (sample size 4631) was with unclear risk of bias. We consider this article to have unclear of bias mainly for two reasons. One is that after 1392 patients had been enrolled, they reduced the TXA dosage from $100 \mathrm{mg} / \mathrm{kg}$ to $50 \mathrm{mg} / \mathrm{kg}$, and the dose reduction underpowered this trial. The other reason is that the first 2127 participants had not been taking aspirin regularly before the trial or had stopped taking aspirin at least 4 days before surgery, while participants who were subsequently enrolled may or may not have been previously exposed to aspirin therapy. Given its large sample size, we did analysis on rest of the studies with low and unclear risk of bias except Myles study, and found that the use of TXA reduced transfusion rate by a relative 27\% (RR 0.73, 95\% CI 0.68 to $0.78, \mathrm{P}<0.00001)$. Excluding the trial from Myles et al. didn't make a difference.

This meta-analysis has several limitations. Some studies only reported transfusion volume without providing data on transfusion rate. We contacted the corresponding authors for missing data, but not much reply was received. Another drawback was that we did not perform a network analysis to compare the effect of high and low-dose regimen, which underpowered our result.

\section{Conclusions}

This meta-analysis provides further evidence that TXA significantly reduces peri-operative blood loss and transfusion requirements in adult cardiac surgery, without increasing the risk of serious adverse events except for seizure. The risk of seizure is only seen in high-dose trials delivered intravenously. High-dose TXA does not further decrease transfusion rate and has a strong tendency to cause more seizure attacks compared to the low-dose TXA. We thus consider low-dose TXA(bolus injection $<50 \mathrm{mg} / \mathrm{kg}$, or $10 \mathrm{mg} / \mathrm{kg}+1 \mathrm{mg} / \mathrm{kg} / \mathrm{h}$ )to be more preferable.

\section{Additional files}

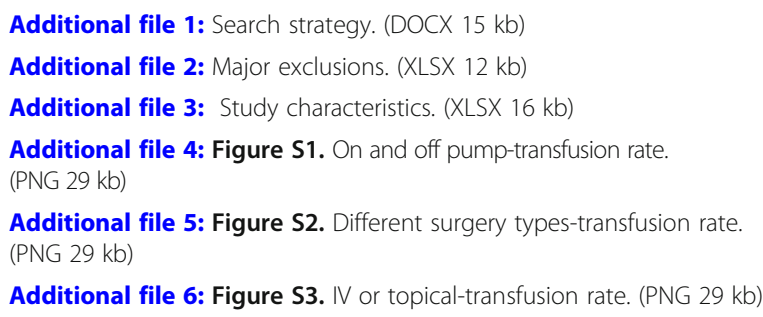

Additional file 7: Figure S4. Transfusion rate-exclude high risk. (PNG $22 \mathrm{~kb}$ )

Additional file 8: Figure S5. Mortality. (PNG $20 \mathrm{~kb}$ )

Additional file 9: Figure S6. PE. (PNG $15 \mathrm{~kb}$ )

Additional file 10: Figure S7. Strole. (PNG $21 \mathrm{~kb}$ )

Additional file 11: Figure S8. MI. (PNG $21 \mathrm{~kb}$ )

Additional file 12: Figure S9. Renal dysfunction. (PNG $16 \mathrm{~kb}$ )

Additional file 13: Figure S10. Funnel Plot for transfusion rate. (PNG $6 \mathrm{~kb}$ )

Abbreviations

CPB: Cardiopulmonary bypass; RCTs: Randomized controlled trials; TXA: Tranexamic acid

\section{Acknowledgements}

We would like to thank doctor Chenghui Zhou in providing methodology guidance for this meta-analysis. We would like to thank doctor Yang Cong and doctor Zefei Zhang for helping with the full-text search process.

\section{Authors' contributions}

JS did the overall design of the meta-analysis. JG and XG searched database for relevant studies. JG and Yan Ma extracted data from the original studies. $\mathrm{HL}, \mathrm{WH}$ and SZ helped with data management. JG and XG were major contributors in writing the manuscript. $\mathrm{HJ}$ and GW helped with writing and the revision process. All authors read and approved the final manuscript.

\section{Funding}

This research did not receive any specific grant from funding agencies in the public, commercial, or not-for-profit sectors.

\section{Availability of data and materials}

The data sets used and/or analyzed during the current study are available from the corresponding author on reasonable request.

\section{Ethics approval and consent to participate}

Not applicable.

\section{Consent for publication}

Not applicable.

\section{Competing interests}

The authors declare that they have no competing interests.

\section{Author details}

'Department of Anesthesiology, Fuwai Hospital, National Center for Cardiovascular Diseases, Chinese Academy of Medical Sciences, and Peking Union Medical College, No.167 Beilishi Road, Xicheng district, Beijing, China. ${ }^{2}$ Department of Blood Transfusion, Fuwai Hospital, National Center for Cardiovascular Diseases, Chinese Academy of Medical Sciences, and Peking Union Medical College, No.167 Beilishi Road, Xicheng district, Beijing, China. ${ }^{3}$ Operating room, Fuwai Hospital, National Center for Cardiovascular Diseases, Chinese Academy of Medical Sciences, and Peking Union Medical College, No.167 Beilishi Road, Xicheng district, Beijing, China. ${ }^{4}$ Department of Anesthesiology, The 305th Hospital of the Chinese People's Liberation Army, No.13 Wenjin Road, Xicheng district, Beijing, China. ${ }^{5}$ Department of Anesthesiology, Wu'an First People's Hospital, Kuangjian Road, Handan, Hebei Province, China.

Received: 16 September 2018 Accepted: 28 May 2019 Published online: 15 July 2019

\section{References}

1. Faught C, Wells P, Fergusson D, Laupacis A. Adverse effects of methods for minimizing perioperative allogeneic transfusion: a critical review of the literature. Transfus Med Rev. 1998;12(3):206-25.

2. Henry DA, Carless PA, Moxey AJ, O'Connell D, Stokes BJ, Fergusson DA, et al. Anti-fibrinolytic use for minimising perioperative allogeneic blood transfusion. Cochrane Database Syst Rev. 2011;(3):Cd001886. 
3. Baharoglu MI, Germans MR, Rinkel GJ, Algra A, Vermeulen M, van Gijn J, et al. Antifibrinolytic therapy for aneurysmal subarachnoid haemorrhage. Cochrane Database Syst Rev. 2013;(8):Cd001245.

4. Ngaage DL, Bland JM. Lessons from aprotinin: is the routine use and inconsistent dosing of tranexamic acid prudent? Meta-analysis of randomised and large matched observational studies. Eur J Cardiothorac Surg. 2010;37(6):1375-83.

5. Murkin JM, Falter F, Granton J, Young B, Burt C, Chu M. High-dose tranexamic acid is associated with nonischemic clinical seizures in cardiac surgical patients. Anesth Analg. 2010;110(2):350-3.

6. Myles PS, Smith JA, Forbes A, Silbert B, Jayarajah M, Painter T, et al. Tranexamic acid in patients undergoing coronary-artery surgery. N Engl J Med. 2017;376(2):136-48

7. Andersson L, Nilsoon IM, Colleen S, Granstrand B, Melander B. Role of urokinase and tissue activator in sustaining bleeding and the management thereof with EACA and AMCA. Ann N Y Acad Sci. 1968;146(2):642-58.

8. Soslau G, Horrow J, Brodsky I. Effect of tranexamic acid on platelet ADP during extracorporeal circulation. Am J Hematol. 1991;38(2):113-9.

9. Grassin-Delyle S, Tremey B, Abe E, Fischler M, Alvarez JC, Devillier P, et al. Population pharmacokinetics of tranexamic acid in adults undergoing cardiac surgery with cardiopulmonary bypass. $\mathrm{Br} J$ Anaesth. 2013;111(6):916-24.

10. Dowd NP, Karski JM, Cheng DC, Carroll JA, Lin Y, James RL, et al. Pharmacokinetics of tranexamic acid during cardiopulmonary bypass. Anesthesiology. 2002;97(2):390-9.

11. Stief TW, Tranexamic acid might stop severe bleeding by intrinsic generation of thrombin. Br Med J. 2012, e-letter available at http:// www.bmj.com/content/345/bmj.e5839/rr/603962

12. Zhang Y, Gao X, Yuan S, Guo J, Lv H, Zhou Y, et al. Effects of tranexamic acid on short-term and long-term outcomes of on-pump coronary artery bypass grafting: randomized trial and 7-year follow-up. 2018;36(6):e12472.

13. Mirmohammadsadeghi A, Mirmohammadsadeghi M, Kheiri M. Does topical tranexamic acid reduce postcoronary artery bypass graft bleeding? J Res Med Sci. 2018;23:6

14. Chaudhary FA, Pervaz Z, llyas S, Niaz MN. Topical use of tranexamic acid in open heart surgery. J Pak Med Assoc. 2018;68(4):538-42.

15. Kimenai DM, Gerritse BM, Lucas C, Rosseel PM, Bentala M, Hattum P, et al. Effectiveness of pericardial lavage with or without tranexamic acid in cardiac surgery patients receiving intravenous tranexamic acid: a randomized controlled trial. Eur J Cardiothorac Surg. 2016;50(6):1124-31 Available from: http://onlinelibrary.wiley.com/o/cochrane/clcentral/articles/ 951/CN-01405951/frame.html.

16. Shah MUA, Asghar MI, Siddiqi R, Chaudhri MS, Janjua AM, labal A. Topical application of tranexamic acid reduces postoperative bleeding in openheart surgery: myth or fact? J Coll Physicians Surg Pak. 2015;25(3):161-5.

17. Hosseini H, Rahimianfar AA, Abdollahi MH, Moshtaghiyoon MM, Haddadzadeh M, Fekri A, et al. Evaluations of topical application of tranexamic acid on post-operative blood loss in off-pump coronary artery bypass surgery. Saudi J Anaesth. 2014;8(2):224-8

18. Alizadeh Ghavidel A, Totonchi Z, Chitsazan M, Dehaki MG, Jalili F, Farsad $F$, et al. Safety and efficacy of caproamin fides and tranexamic acid versus placebo in patients undergoing coronary artery revascularization. J Cardiovasc Thorac Res. 2014;6(3):197-202.

19. Shi J, Ji H, Ren F, Wang G, Xu M, Xue Y, et al. Protective effects of tranexamic acid on clopidogrel before coronary artery bypass grafting: a multicenter randomized trial. JAMA Surg. 2013;148(6):538-47.

20. Nouraei M, Gholipour Baradari A, Ghafari R, Habibi MR, Emami Zeydi A, Sharifi N. Decreasing blood loss and the need for transfusion after CABG surgery: a double-blind randomized clinical trial of topical tranexamic acid. Turk J Med Sci. 2013;43(2):273-8.

21. Esfandiari BR, Bistgani MM, Kabiri M. Low dose tranexamic acid effect on post-coronary artery bypass grafting bleeding. Asian Cardiovasc Thorac Ann. 2013;21(6):669-74 Available from: http://onlinelibrary.wiley.com/o/cochrane/ clcentral/articles/410/CN-00911410/frame.html.

22. Wang $G$, Xie G, Jiang $T$, Wang $Y$, Wang $W$, Ji H, et al. Tranexamic acid reduces blood loss after off-pump coronary surgery: a prospective, randomized, double-blind, placebo-controlled study. Anesth Analg. 2012;115(2):239-43 Available from: http://onlinelibrary.wiley.com/o/ cochrane/clcentral/articles/661/CN-00872661/frame.html.

23. Hassani E, Mahoori A, Mehdizadeh H, Noroozinia H, Aghdashi MM, Saeidi M. The effects of tranexamic acid on postoperative bleeding in coronary artery bypass graft surgery. Tehran Univ Med J. 2012;70(3):176-82.
24. Greiff G, Stenseth R, Wahba A, Videm V, Lydersen S, Irgens W, et al. Tranexamic acid reduces blood transfusions in elderly patients undergoing combined aortic valve and coronary artery bypass graft surgery: a randomized controlled trial. J Cardiothorac Vasc Anesth. 2012;26(2):232-8 Available from: http://onlinelibrary.wiley.com/o/cochrane/clcentral/articles/ 415/CN-00882415/frame.html.

25. Aoki M, Okawa Y, Goto Y, Ogawa S, Baba H. Local administration of tranexamic acid in off-pump coronary artery bypass. Asian Cardiovasc Thorac Ann. 2012;20(6):658-62.

26. Ahn SW, Shim JK, Youn YN, Song JW, Yang SY, Chung SC, et al. Effect of tranexamic acid on transfusion requirement in dual antiplatelet-treated anemic patients undergoing off-pump coronary artery bypass graft surgerya randomized controlled study. Circ J. 2012;76(1):96-101.

27. Hashemi J, Ghaffari Nejad MH, Baharestani B, Esfandiari R, Panahipoor A. Evaluation and comparison of use of low-dose aprotinin and tranexamic acid in CABG: a double-blind, prospective, randomized study of 150 patients. Iran Heart J. 2011;12(1):40-4.

28. Baric D, Unic D, Rudez I, Bacic-Vrca V, Planinc M, Jonjic D, et al. Systemic usage of tranexamic acid is superior to topical: randomized placebocontrolled trial. Interact Cardiovasc Thorac Surg. 2011;12:S92.

29. Taghaddomi RJ, Mirzaee A, Attar AS, Shirdel A. Tranexamic acid reduces blood loss in off-pump coronary artery bypass surgery. J Cardiothorac Vasc Anesth. 2009;23(3):312-5.

30. Later AFL, Maas JJ, Engbers FHM, Versteegh MIM, Bruggemans EF, Dion RAE, et al. Tranexamic acid and aprotinin in low- and intermediate-risk cardiac surgery: a non-sponsored, double-blind, randomised, placebo-controlled trial. Eur J Cardiothorac Surg. 2009;36(2):322-9.

31. Fawzy H, Elmistekawy E, Bonneau D, Latter D, Errett L. Can local application of tranexamic acid reduce post-coronary bypass surgery blood loss? A randomized controlled trial. J Cardiothorac Surg. 2009;4:25.

32. Maddali MM, Rajakumar MC. Tranexamic acid and primary coronary artery bypass surgery: a prospective study. Asian Cardiovasc Thorac Ann. 2007;15(4):313-9.

33. Baric D, Biocina B, Unic D, Sutlic Z, Rudez I, Vrca VB, et al. Topical use of antifibrinolytic agents reduces postoperative bleeding: a double-blind, prospective, randomized study. Eur J Cardiothorac Surg. 2007:31(3):366-71 discussion 71

34. Murphy GJ, Mango E, Lucchetti V, Battaglia F, Catapano D, Rogers CA et al. A randomized trial of tranexamic acid in combination with cell salvage plus a meta-analysis of randomized trials evaluating tranexamic acid in off-pump coronary artery bypass grafting. J Thorac Cardiovasc Surg. 2006;132(3):475-80 80.e1-8.

35. Mehr-Aein A, Sadeghi M, Madani-Civi M. Does tranexamic acid reduce blood loss in off-pump coronary artery bypass? Acta Med Iran. 2006; 44(5):309-15.

36. Abul-Azm A, Abdullah KM. Effect of topical tranexamic acid in open heart surgery. Eur J Anaesthesiol. 2006;23(5):380-4.

37. Diprose P, Herbertson MJ, O'Shaughnessy D, Deakin CD, Gill RS. Reducing allogeneic transfusion in cardiac surgery: a randomized double-blind placebo-controlled trial of antifibrinolytic therapies used in addition to intraoperative cell salvage. Br J Anaesth. 2005;94(3):271-8.

38. Casati V, Della Valle P, Benussi S, Franco A, Gerli C, Baili P, et al. Effects of tranexamic acid on postoperative bleeding and related hematochemical variables in coronary surgery: comparison between on-pump and off-pump techniques. J Thorac Cardiovasc Surg. 2004;128(1):83-91.

39. Andreasen JJ, Nielsen C. Prophylactic tranexamic acid in elective, primary coronary artery bypass surgery using cardiopulmonary bypass. Eur J Cardiothorac Surg. 2004;26(2):311-7.

40. Pleym H, Stenseth R, Wahba A, Bjella L, Karevold A, Dale O. Single-dose tranexamic acid reduces postoperative bleeding after coronary surgery in patients treated with aspirin until surgery. Anesth Analg. 2003;96(4):923-8 table of contents.

41. Jares M, Vanek T, Straka Z, Brucek P. Tranexamic acid reduces bleeding after off-pump coronary artery bypass grafting. J Cardiovasc Surg. 2003;44(2):205-8.

42. Zabeeda D, Medalion B, Sverdlov M, Ezra S, Schachner A, Ezri T, et al. Tranexamic acid reduces bleeding and the need for blood transfusion in primary myocardial revascularization. Ann Thorac Surg. 2002;74(3):733-8.

43. Uozaki Y, Watanabe G, Kotou K, Ueyama K, Doi Y, Misaki T. Effect of tranexamic acid on blood loss reduction after cardiopulmonary bypass. Jpn J Thorac Cardiovasc Surg = Nihon Kyobu Geka Gakkai zasshi. 2001;49(5):273-8. 
44. Kamada K, Yamakage M, Niiya T, Tsujiguchi N, Chen X, Namiki A. Celite-activated viscometer Sonoclot can measure the suppressive effect of tranexamic acid on hyperfibrinolysis in cardiac surgery. J Anesth. 2001;15(1):17-21.

45. Casati V, Gerli C, Franco A, Torri G, D'Angelo A, Benussi S, et al. Tranexamic acid in off-pump coronary surgery: a preliminary, randomized, double-blind, placebo-controlled study. Ann Thorac Surg. 2001;72(2):470-5.

46. Armellin G, Casella S, Guzzinati S, Pasini L, Marcassa A, Giron G. Tranexamic acid in aortic valve replacement. J Cardiothorac Vasc Anesth. 2001;15(3):331-5.

47. De Bonis M, Cavaliere F, Alessandrini F, Lapenna E, Santarelli F, Moscato U, et al. Topical use of tranexamic acid in coronary artery bypass operations: a double-blind, prospective, randomized, placebo-controlled study. J Thorac Cardiovasc Surg. 2000;119(3):575-80.

48. Misfeld M, Dubbert S, Eleftheriadis S, Siemens HJ, Wagner T, Sievers HH. Fibrinolysis-adjusted perioperative low-dose aprotinin reduces blood loss in bypass operations. Ann Thorac Surg. 1998;66(3):792-9.

49. Hardy JF, Bélisle S, Dupont C, Harel F, Robitaille D, Roy M, et al. Prophylactic tranexamic acid and epsilon-aminocaproic acid for primary myocardial revascularization. Ann Thorac Surg. 1998;65(2):371-6.

50. Pinosky ML, Kennedy DJ, Fishman RL, Reeves ST, Alpert CC, Ecklund J, et al. Tranexamic acid reduces bleeding after cardiopulmonary bypass when compared to epsilon aminocaproic acid and placebo. J Card Surg. 1997;12(5):330-8.

51. Landymore RW, Murphy JT, Lummis H, Carter C. The use of low-dose aprotinin, epsilon-aminocaproic acid or tranexamic acid for prevention of mediastinal bleeding in patients receiving aspirin before coronary artery bypass operations. Eur J Cardiothorac Surg. 1997;11(4):798-800.

52. Brown RS, Thwaites BK, Mongan PD. Tranexamic acid is effective in decreasing postoperative bleeding and transfusions in primary coronary artery bypass operations: a double-blind, randomized, placebo-controlled trial. Anesth Analg. 1997;85(5):963-70.

53. Shore-Lesserson L, Reich DL, Vela-Cantos F, Ammar T, Ergin MA. Tranexamic acid reduces transfusions and mediastinal drainage in repeat cardiac surgery. Anesth Analg. 1996;83(1):18-26.

54. Katsaros D, Petricevic M, Snow NJ, Woodhall DD, Van Bergen R. Tranexamic acid reduces postbypass blood use: a double-blinded, prospective, randomized study of 210 patients. Ann Thorac Surg. 1996;61(4):1131-5.

55. Speekenbrink RGH, Vonk ABA, Wildevuur CRH, Eijsman L. Hemostatic efficacy of dipyridamole, tranexamic acid, and aprotinin in coronary bypass grafting. Ann Thorac Surg. 1995;59(2):438-42.

56. Rousou JA, Engelman RM, Flack JE, Deaton DW, Owen SG. Tranexamic acid significantly reduces blood loss associated with coronary revascularization. Ann Thorac Surg. 1995;59(3):671-5

57. Pugh SC, Wielogorski AK. A comparison of the effects of tranexamic acid and low-dose aprotinin on blood loss and homologous blood usage in patients undergoing cardiac surgery. J Cardiothorac Vasc Anesth. 1995;9(3):240-4.

58. De Peppo AP, Pierri MD, Scafuri A, De Paulis R, Colantuono G, Caprara $E$, et al. Intraoperative antifibrinolysis and blood-saving techniques in cardiac surgery: prospective trial of 3 antifibrinolytic drugs. Tex Heart Inst J. 1995;22(3):231-6.

59. Ali IM, Landymore RW. The use of tranexamic acid in cardiac operations [10]. J Thorac Cardiovasc Surg. 1994;107(5):1377.

60. Couture P, Lebon JS, Laliberte E, Desjardins G, Chamberland ME, Ayoub C, et al. Low-dose versus high-dose tranexamic acid reduces the risk of nonischemic seizures after cardiac surgery with cardiopulmonary bypass. J Cardiothorac Vasc Anesth. 2017;31(5):1611-7.

61. Takagi H, Ando T, Umemoto T. Seizures associated with tranexamic acid for cardiac surgery: a meta-analysis of randomized and non-randomized studies. J Cardiovasc Surg. 2017;58(4):633-41.

62. Taksaudom N, Siwachat S, Tantraworasin A. Additional effects of topical tranexamic acid in on-pump cardiac surgery. Asian Cardiovasc Thorac Ann. 2017;25(1):24-30 Available from: http://onlinelibrary.wiley.com/o/cochrane/ clcentral/articles/737/CN-01329737/frame.html.

63. Spegar J, Vanek T, Snircova J, Fajt R, Straka Z, Pazderkova P, et al. Local and systemic application of tranexamic acid in heart valve surgery: a prospective, randomized, double blind LOST study. J Thromb Thrombolysis. 2011;32(3):303-10.

64. Imtiaz A, Mujahid ul I, Ansa I, Azmat Ali S. Effects of bolus dose and continuous infusion of tranexamic acid on blood loss after coronary artery bypass grafting. J Ayub Med Coll Abbottabad 2014;26(3):371-375.
65. Dincq AS, Michaux I, Lacrosse D, Collard E, Jamart J. High-dose versus lowdose of tranexamic acid in cardiac surgical patients receiving aspirin until surgery. J Cardiothorac Vasc Anesth. 2010;24(3):S36.

66. Du Y, Xu J, Wang G, Shi J, Yang L, Shi S, et al. Comparison of two tranexamic acid dose regimens in patients undergoing cardiac valve surgery. J Cardiothorac Vasc Anesth. 2014;28(5):1233-7.

67. Jiménez JJ, Iribarren JL, Brouard M, Hernández D, Palmero S, Jiménez A, et al. Safety and effectiveness of two treatment regimes with tranexamic acid to minimize inflammatory response in elective cardiopulmonary bypass patients: a randomized double-blind, dose-dependent, phase IV clinical trial. J Cardiothorac Surg. 2011;6:138.

68. Karski JM, Dowd NP, Joiner R, Carroll J, Peniston C, Bailey K, et al. The effect of three different doses of tranexamic acid on blood loss after cardiac surgery with mild systemic hypothermia (32 degrees C). J Cardiothorac Vasc Anesth. 1998;12(6):642-6.

69. Lambert W, Brisebois FJ, Wharton TJ, Carrier RC, Boyle D, Rowe BH. The effectiveness of low dose tranexamic acid in primary cardiac surgery. Can J Anaest $=$ Journal canadien d'anesthesie. 1998;45(6):571-4.

70. Sigaut S, Tremey B, Ouattara A, Couturier R, Taberlet C, Grassin-Delyle S, et al. Comparison of two doses of tranexamic acid in adults undergoing cardiac surgery with cardiopulmonary bypass. Anesthesiology. 2014;120(3):590-600.

71. Karski JM, Teasdale SJ, Norman P, Carroll J, VanKessel K, Wong P, et al. Prevention of bleeding after cardiopulmonary bypass with high-dose tranexamic acid. Double-blind, randomized clinical trial. J Thorac Cardiovasc Surg. 1995;110(3):835-42.

72. Waldow T, Szlapka M, Haferkorn M, Bürger L, Plötze K, Matschke K. Prospective clinical trial on dosage optimizing of tranexamic acid in non-emergency cardiac surgery procedures. Clin Hemorheol Microcirc. 2013;55(4):457-68

73. Sharma V, Fan J, Jerath A, Pang KS, Bojko B, Pawliszyn J, et al. Pharmacokinetics of tranexamic acid in patients undergoing cardiac surgery with use of cardiopulmonary bypass. Anaesthesia. 2012;67(11):1242-50.

\section{Publisher's Note}

Springer Nature remains neutral with regard to jurisdictional claims in published maps and institutional affiliations.

Ready to submit your research? Choose BMC and benefit from:

- fast, convenient online submission

- thorough peer review by experienced researchers in your field

- rapid publication on acceptance

- support for research data, including large and complex data types

- gold Open Access which fosters wider collaboration and increased citations

- maximum visibility for your research: over $100 \mathrm{M}$ website views per year

At BMC, research is always in progress.

Learn more biomedcentral.com/submissions 